\title{
An Extension of Kedlaya's Algorithm to Hyperelliptic Curves in Characteristic 2
}

\author{
Jan Denef $^{1}$ and Frederik Vercauteren ${ }^{2,3}$ \\ 1 Department of Mathematics \\ University of Leuven \\ Celestijnenlaan 200B, B-3001 Leuven-Heverlee, Belgium \\ jan.denef@wis. kuleuven. ac . be \\ 2 Department of Electrical Engineering \\ University of Leuven \\ Kasteelpark Arenberg 10, B-3001 Leuven-Heverlee, Belgium \\ frederik.vercauteren@esat.kuleuven.ac.be \\ ${ }^{3}$ Computer Science Department \\ University of Bristol \\ Woodland Road, Bristol BS8 1UB, United Kingdom \\ frederik@cs.bris.ac.uk
}

\begin{abstract}
We present an algorithm to compute the zeta function of an arbitrary hyperelliptic curve over a finite field $\mathbb{F}_{q}$ of characteristic 2 , thereby extending the algorithm of Kedlaya for odd characteristic. Given a genus $g$ hyperelliptic curve defined over $\mathbb{F}_{2^{n}}$, the average-case time complexity is $O\left(g^{4+\varepsilon} n^{3+\varepsilon}\right)$ and the average-case space complexity is $O\left(g^{3} n^{3}\right)$, whereas the worst-case time and space complexities are $O\left(g^{5+\varepsilon} n^{3+\varepsilon}\right)$ and $O\left(g^{4} n^{3}\right)$ respectively.
\end{abstract}

Keywords: Hyperelliptic curves, cryptography, Kedlaya's algorithm, MonskyWashnitzer cohomology

\section{Introduction}

Since elliptic curve cryptosystems were introduced by Koblitz [18] and Miller [27], various other systems based on the discrete logarithm problem in the Jacobian of curves have been proposed such as hyperelliptic curves [19], superelliptic curves [12] and $\mathcal{C}_{a b}$ curves [2]. One of the main initialization steps of these cryptosystems is to generate a suitable curve defined over a given finite field. To ensure the security of the system, the curve must be chosen such that the group order of the Jacobian is divisible by a large prime.

Currently, there exist several approaches for computing the number of points on the Jacobian of curves. The first method is $l$-adic in nature: the number of points is computed modulo sufficient small primes $l$ by working in $l$-torsion subgroups of the Jacobian and the final result is determined using the Chinese remainder theorem. This approach was first described by Schoof [36] for elliptic 
curves and leads to a polynomial time algorithm in all characteristics. A detailed description of Schoof's algorithm and the improvements by Atkin [3] and Elkies [9] can be found in [4] and [23]. Pila [33] and later Adleman and Huang [1] extended Schoof's algorithm to higher genus curves. Currently, only the genus 2 version of this algorithm is practical $[14,15]$.

The second approach is $p$-adic in nature and is especially efficient for algebraic varieties over finite fields of small characteristic. These $p$-adic algorithms come in two flavours. The first strategy computes a $p$-adic approximation of the SerreTate canonical lift and the action of Frobenius on this lift. This approach was first described by Satoh [34] for elliptic curves. An overview of the many variants and further optimizations of Satoh's algorithm can be found in [39]. Mestre [25] presented a "dual" algorithm using the Arithmetic-Geometric mean and sketched how it could be extended to ordinary hyperelliptic curves [26]. Results by Lercier and Lubicz [24] show that this algorithm is very efficient as long as the genus is small; this is due to the exponential dependence on the genus.

The second strategy computes the action of Frobenius on $p$-adic cohomology groups. Kedlaya [17] described such an algorithm for hyperelliptic curves over finite fields of small odd characteristic, using the theory of Monsky-Washnitzer cohomology. The running time of the algorithm is $O\left(g^{4+\varepsilon} n^{3+\varepsilon}\right)$ for a hyperelliptic curve of genus $g$ over $\mathbb{F}_{p^{n}}$. The algorithm readily generalizes to superelliptic curves as shown by Gaudry and Gürel [13]. A related approach by Lauder and Wan [20] is based on Dwork's proof of the rationality of the zeta function and results in a polynomial time algorithm to compute the zeta function of an arbitrary algebraic variety over a finite field. Despite its polynomial time complexity, a first implementation indicates that cryptographical sizes are out of reach. Using Dwork cohomology, Lauder and Wan [21,22] specialised their original algorithm to Artin-Schreier curves, leading to an $O\left(g^{5+\varepsilon} n^{3+\varepsilon}\right)$ time algorithm. In [7], we described an extension of Kedlaya's algorithm to Artin-Schreier curves in characteristic 2 with the same time complexity.

In this paper we extend Kedlaya's algorithm to arbitrary hyperelliptic curves defined over a finite field of characteristic 2. Given a genus $g$ hyperelliptic curve defined over $\mathbb{F}_{2^{n}}$, the average-case time complexity is $O\left(g^{4+\varepsilon} n^{3+\varepsilon}\right)$ and the average-case space complexity is $O\left(g^{3} n^{3}\right)$, whereas the worst-case time and space complexities are $O\left(g^{5+\varepsilon} n^{3+\varepsilon}\right)$ and $O\left(g^{4} n^{3}\right)$ respectively. An implementation in the $\mathrm{C}$ programming language shows that cryptographical sizes are now feasible for any genus $g$. This paper is the theoretical version of [38]: it provides a detailed description of the underlying mathematics, presents all missing proofs and corrects the complexity analysis.

The remainder of the paper is organized as follows: Section 2 reviews the basics of Monsky-Washnitzer cohomology and Section 3 shows how to extend Kedlaya's algorithm to characteristic 2 . Section 4 contains a ready to implement description of the resulting algorithm and a detailed complexity analysis. Finally, Section 5 presents running times and memory usages of an implementation in the $\mathrm{C}$ programming language. 


\section{Monsky-Washnitzer Cohomology}

In this section we briefly recall the definition of Monsky-Washnitzer cohomology as introduced by Monsky and Washnitzer [29-31]; more details can be found in the lectures by Monsky [32] and the survey by van der Put [37].

Let $\bar{X}$ be a smooth affine variety over a finite field $k:=\mathbb{F}_{q}$ with $q=p^{n}$ elements. Denote the coordinate ring of $\bar{X}$ by $\bar{A}$. Let $R$ be the ring of Witt vectors of $\mathbb{F}_{q}$, i.e. the degree $n$ unramified extension of the $p$-adic integers $\mathbb{Z}_{p}$ with residue field $\mathbb{F}_{q}$ and let $K$ be the fraction field of $R$. Elkik [10] showed that there always exists a smooth finitely generated $R$-algebra $A$ such that $A \otimes_{R} \mathbb{F}_{q} \cong \bar{A}$. In general $A$ does not allow a lift of the Frobenius endomorphism $\bar{F}$ on $\bar{A}$; Monsky and Washnitzer solve this problem by constructing a subalgebra $A^{\dagger}$ of the $p$-adic completion of $A$, whose elements satisfy growth conditions. The dagger ring or weak completion $A^{\dagger}$ is defined as follows: write $A:=R\left[x_{1}, \ldots, x_{n}\right] /\left(f_{1}, \ldots, f_{m}\right)$, then

$$
A^{\dagger}:=R\left\langle x_{1}, \ldots, x_{n}\right\rangle^{\dagger} /\left(f_{1}, \ldots, f_{m}\right),
$$

where $R\left\langle x_{1}, \ldots, x_{n}\right\rangle^{\dagger}$ consists of power series

$$
\left\{\sum a_{\alpha} x^{\alpha} \in R\left[\left[x_{1}, \ldots, x_{n}\right]\right]|\exists C, \rho \in \mathbb{R}, C>0,0<\rho<1, \forall \alpha:| a_{\alpha} \mid \leq C \rho^{|\alpha|}\right\}
$$

with $\alpha:=\left(\alpha_{1}, \ldots, \alpha_{n}\right), x^{\alpha}:=x_{1}^{\alpha_{1}} \cdots x_{n}^{\alpha_{n}}$ and $|\alpha|:=\sum_{i=0}^{n} \alpha_{i}$.

Let $\bar{B} / k$ and $B / R$ be smooth and finitely generated with $B \otimes_{R} \mathbb{F}_{q} \cong \bar{B}$ and let $B^{\dagger}$ be the dagger ring of $B$. Given a morphism of $k$-algebra's $\bar{G}: \bar{A} \rightarrow \bar{B}$, there always exists an $R$-morphism $G: A^{\dagger} \rightarrow B^{\dagger}$ lifting $\bar{G}$. This last property implies that we can lift the $q$-power Frobenius from $\bar{A}$ to $A^{\dagger}$.

For $A^{\dagger}$ we can define the universal module $D^{1}\left(A^{\dagger}\right)$ of differentials

$$
D^{1}\left(A^{\dagger}\right):=\left(A^{\dagger} d x_{1}+\cdots+A^{\dagger} d x_{n}\right) /\left(\sum_{i=1}^{m} A^{\dagger}\left(\frac{\partial f_{i}}{\partial x_{1}} d x_{1}+\cdots+\frac{\partial f_{i}}{\partial x_{n}} d x_{n}\right)\right)
$$

Let $D^{i}\left(A^{\dagger}\right):=\bigwedge^{i} D^{1}\left(A^{\dagger}\right)$ be the $i$-th exterior product of $D^{1}\left(A^{\dagger}\right)$ and denote with $d_{i}: D^{i}\left(A^{\dagger}\right) \rightarrow D^{i+1}\left(A^{\dagger}\right)$ the exterior differentiation. Since $d_{i+1} \circ d_{i}=0$ we get the de Rham complex $D\left(A^{\dagger}\right)$

$$
0 \longrightarrow D^{0}\left(A^{\dagger}\right) \stackrel{d_{0}}{\longrightarrow} D^{1}\left(A^{\dagger}\right) \stackrel{d_{1}}{\longrightarrow} D^{2}\left(A^{\dagger}\right) \stackrel{d_{2}}{\longrightarrow} D^{3}\left(A^{\dagger}\right) \cdots
$$

The $i$-th cohomology group of $D\left(A^{\dagger}\right)$ is defined as $H^{i}(\bar{A} / R):=\operatorname{Ker} d_{i} / \operatorname{Im} d_{i-1}$ and $H^{i}(\bar{A} / K):=H^{i}(\bar{A} / R) \otimes_{R} K$ finally defines the $i$-th Monsky-Washnitzer cohomology group. One can prove that for smooth, finitely generated $k$-algebra's $\bar{A}$ the map $\bar{A} \mapsto H^{\bullet}(\bar{A} / K)$ is well defined and functorial, which justifies the notation. Replacing $A^{\dagger}$ with $A$ in the above construction of the $i$-th MonskyWashnitzer cohomology group $H^{i}(\bar{A} / K)$ gives rise to the $i$-th algebraic de Rham cohomology group $H_{D R}^{i}(A / K)$. Unlike the Monsky-Washnitzer cohomology, the algebraic de Rham cohomology essentially depends on the algebra $A$ and in general $H^{i}(\bar{A} / K)$ will not be isomorphic to $H_{D R}^{i}(A / K)$. 
Let $F$ be a lift of the $q$-power Frobenius endomorphism of $\bar{A}$ to $A^{\dagger}$, then $F$ induces an endomorphism $F_{*}$ on the cohomology groups $H^{i}(\bar{A} / K)$. The main theorem of Monsky-Washnitzer cohomology is that these groups satisfy a Lefschetz fixed point formula.

Theorem 1 (Lefschetz fixed point formula) Let $\bar{X} / \mathbb{F}_{q}$ be a smooth affine variety of dimension $d$, then the number of $\mathbb{F}_{q}$-rational points on $\bar{X}$ equals

$$
\sum_{i=0}^{d}(-1)^{i} \operatorname{Tr}\left(q^{d} F_{*}^{-1} \mid H^{i}(\bar{A} / K)\right) \text {. }
$$

\section{Cohomology of Hyperelliptic Curves}

\subsection{Overview of Kedlaya's Construction}

Let $\mathbb{F}_{q}$ be a finite field with $q=p^{n}$ elements and fix an algebraic closure $\overline{\mathbb{F}}_{q}$. Throughout this section we will assume that $p$ is a small odd prime. Let $\bar{Q}(x)$ be a monic polynomial of degree $2 g+1$ over $\mathbb{F}_{q}$ without repeated roots and let $\bar{C}$ be the affine hyperelliptic curve defined by the equation $y^{2}=\bar{Q}(x)$. Kedlaya does not work with the curve $\bar{C}$ itself, but with the affine curve $\bar{C}^{\prime}$ which is obtained from $\bar{C}$ by removing the locus of $y=0$, i.e. the points $\left(\bar{\xi}_{i}, 0\right) \in \overline{\mathbb{F}}_{q} \times \overline{\mathbb{F}}_{q}$ where $\bar{\xi}_{i}$ is a zero of $\bar{Q}(x)$. The coordinate ring $\bar{A}$ of $\bar{C}^{\prime}$ is clearly given by $\mathbb{F}_{q}\left[x, y, y^{-1}\right] /\left(y^{2}-\bar{Q}(x)\right)$.

Let $K$ be a degree $n$ unramified extension of $\mathbb{Q}_{p}$, with valuation ring $R$, such that $R / p R=\mathbb{F}_{q}$. Take any monic lift $Q(x) \in R[x]$ of $\bar{Q}(x)$ and let $C$ be the smooth affine hyperelliptic curve defined by $y^{2}=Q(x)$. Let $C^{\prime}$ be the curve obtained from $C$ by removing the locus of $y=0$. Then the coordinate ring of $C^{\prime}$ is $A=R\left[x, y, y^{-1}\right] /\left(y^{2}-Q(x)\right)$. Let $A^{\dagger}$ denote the weak completion of $A$. Since $\bar{F}=\bar{\sigma}^{n}$, with $\bar{\sigma}$ the $p$-power Frobenius, it is sufficient to lift $\bar{\sigma}$ to an endomorphism $\sigma$ of $A^{\dagger}$. It is natural to define $\sigma$ as the Frobenius substitution on $R$ and to extend it to $A^{\dagger}$ by mapping $x$ to $x^{\sigma}:=x^{p}$ and $y$ to $y^{\sigma}$ with

$$
y^{\sigma}:=y^{p}\left(1+\frac{Q(x)^{\sigma}-Q(x)^{p}}{Q(x)^{p}}\right)^{1 / 2}=y^{p} \sum_{i=0}^{\infty}\left(\begin{array}{c}
1 / 2 \\
i
\end{array}\right) \frac{\left(Q(x)^{\sigma}-Q(x)^{p}\right)^{i}}{y^{2 p i}} .
$$

An easy calculation shows that $\operatorname{ord}_{p}\left(\begin{array}{c}1 / 2 \\ i\end{array}\right) \geq 0$ which implies that $y^{\sigma}$ is an element of $A^{\dagger}$ since $p$ divides $Q(x)^{\sigma}-Q(x)^{p}$. Note that it is essential that $y^{-1}$ is an element of $A^{\dagger}$, which explains why we compute with $C^{\prime}$ instead of $C$.

Since $C^{\prime}$ has dimension one, the only non-trivial Monsky-Washnitzer cohomology groups are $H^{0}(\bar{A} / K)$ and $H^{1}(\bar{A} / K)$. Finding a basis for $H^{0}(\bar{A} / K)$ is easy since by definition $H^{0}(\bar{A} / K):=\operatorname{Ker} d_{0}$, with $d_{0}$ the derivation from $A^{\dagger}$ into $D^{1} A^{\dagger}$, which implies that $H^{0}(\bar{A} / K)$ is a one dimensional $K$-vectorspace. The case $H^{1}(\bar{A} / K)$ is more difficult and proceeds in two steps. Kedlaya first constructs a basis for the algebraic de Rham cohomology of $A$ and devises reduction formulae to express any differential form on this basis. Then he proves 
that these formulae lead to a convergent process when applied to the de Rham cohomology of $A^{\dagger}$, i.e. $H^{1}(\bar{A} / K)$ and concludes that the basis for the algebraic de Rham cohomology also is a basis for $H^{1}(\bar{A} / K)$.

The de Rham cohomology of $A$ splits into eigenspaces under the hyperelliptic involution: a positive eigenspace generated by $x^{i} / y^{2} d x$ for $i=0, \ldots, 2 g$ and a negative eigenspace generated by $x^{i} / y d x$ for $i=0, \ldots, 2 g-1$. Using the equation of the curve, any differential form can be written as $\sum_{k=-B_{U}}^{B_{L}} \sum_{i=0}^{2 g} a_{i, k} x^{i} / y^{k} d x$ with $a_{i, k} \in K$ and $B_{U}, B_{L} \in \mathbb{N}$. A differential of the form $P(x) / y^{s} d x$ with $P(x) \in K[x]$ and $s \in \mathbb{N}$ can be reduced as follows. Since $Q(x)$ has no repeated roots, we can always write the polynomial $P(x)=U(x) Q(x)+V(x) Q^{\prime}(x)$. Using the fact that $d\left(V(x) / y^{s-2}\right)$ is exact, one obtains

$$
\frac{P(x)}{y^{s}} d x \equiv\left(U(x)+\frac{2 V^{\prime}(x)}{(s-2)}\right) \frac{d x}{y^{s-2}},
$$

where $\equiv$ means equality modulo exact differentials. This congruence can be used to reduce a differential form involving negative powers of $y$ to the case $s=1$ and $s=2$. A differential $P(x) / y d x$ with $\operatorname{deg} P=m \geq 2 g$ can be reduced by repeatedly subtracting suitable multiples of the exact differential $d\left(x^{i-2 g} y\right)$ for $i=m, \ldots, 2 g$. Finally, it is clear that the differential $P(x) / y^{2} d x$ is congruent to $(P(x) \bmod Q(x)) / y^{2} d x$ modulo exact differentials. A differential of the form $P(x) y^{s} d x$ with $P(x) \in K[x]$ and $s \in \mathbb{N}$ is exact if $s$ is even and equal to $P(x) Q(x)^{\lceil s / 2\rceil} / y d x$ if $s$ is odd and thus can be reduced using the above reduction formula.

Kedlaya then proves two lemmata which bound the denominators introduced during the above reduction process. The result is as follows: let $A(x) \in R[x]$ be a polynomial of degree $\leq 2 g$, then for $k \in \mathbb{N}$ the reduction of $A(x) y^{2 k+1} d x$ becomes integral upon multiplication by $p^{\left\lfloor\log _{p}((2 g+1)(k+1)-2)\right\rfloor}$ and the reduction of $A(x) / y^{2 k+1} d x$ becomes integral upon multiplication by $p^{\left\lfloor\log _{p}(2 k+1)\right\rfloor}$. This implies that the reduction process converges for elements of $D^{1}\left(A^{\dagger}\right)$.

The final step in the algorithm consists of computing the action induced by $\sigma$ on a basis of $H^{1}(\bar{A} / K)$. Using the Lefschetz fixed point theorem, Kedlaya shows that it is sufficient to compute the matrix $M$ through which $\sigma$ acts on the antiinvariant part $H^{1}(\bar{A} / K)^{-}$of $H^{1}(\bar{A} / K)$. Therefore we only need to compute $\left(x^{i} / y d x\right)^{\sigma}=p x^{p(i+1)-1} / y^{\sigma} d x$ for $i=0, \ldots, 2 g-1$. Using the aforementioned reduction process we express $\left(x^{i} / y d x\right)^{\sigma}$ on the basis of $H^{1}(\bar{A} / K)^{-}$and compute the matrix $M$. The characteristic polynomial of Frobenius can then be recovered from the coefficients of the characteristic polynomial of the matrix $M M^{\sigma} \cdots M^{\sigma^{n-1}}$ through which the Frobenius $F=\sigma^{n}$ acts on $H^{1}(\bar{A} / K)^{-}$.

\subsection{Cohomology of Hyperelliptic Curves over $\mathbb{F}_{2^{n}}$}

Let $\mathbb{F}_{q}$ be a finite field with $q=2^{n}$ elements and fix an algebraic closure $\overline{\mathbb{F}}_{q}$. Consider the smooth affine hyperelliptic curve $\bar{C}$ of genus $g$ defined by the equation

$$
\bar{C}: y^{2}+\bar{h}(x) y=\bar{f}(x),
$$


with $\bar{h}(x), \bar{f}(x) \in \mathbb{F}_{q}[x], \bar{f}(x)$ monic of degree $2 g+1$ and $\operatorname{deg} \bar{h} \leq g$. Write $\bar{h}(x)$ as $\bar{c} \cdot \prod_{i=0}^{s}\left(x-\bar{\theta}_{i}\right)^{m_{i}}$ with $\bar{\theta}_{i} \in \overline{\mathbb{F}}_{q}, \bar{c} \in \mathbb{F}_{q} \backslash\{0\}$ the leading coefficient of $\bar{h}(x)$ and define $\bar{H}(x)=\prod_{i=0}^{s}\left(x-\bar{\theta}_{i}\right) \in \mathbb{F}_{q}[x]$. If $h(x)$ is a constant, we set $\bar{H}(x)=1$. Without loss of generality we can assume that $\bar{H}(x) \mid \bar{f}(x)$. Indeed, the isomorphism defined by $x \mapsto x$ and $y \mapsto y+\sum_{i=0}^{s} \bar{b}_{i} x^{i}$ transforms the curve in

$$
y^{2}+\bar{h}(x) y=\bar{f}(x)-\sum_{i=0}^{s} \bar{b}_{i}^{2} x^{2 i}-\bar{h}(x) \sum_{i=0}^{s} \bar{b}_{i} x^{i} .
$$

The polynomial $\bar{H}(x)$ will divide the right hand side of the above equation if and only if $\bar{f}\left(\bar{\theta}_{j}\right)=\sum_{i=0}^{s} \bar{b}_{i}^{2} \cdot \bar{\theta}_{j}^{2 i}$ for $j=0, \ldots, s$. This is a system of linear equations in the indeterminates $\bar{b}_{i}^{2}$ and its determinant is a Vandermonde determinant. Since the $\bar{\theta}_{j}$ are the zeros of a polynomial defined over $\mathbb{F}_{q}$, the system of equations is invariant under the $q$-power Frobenius automorphism $\bar{F}$ and it follows that the $\bar{b}_{i}^{2}$ and therefore the $\bar{b}_{i}$ are elements of $\mathbb{F}_{q}$. We conclude that we can always assume that $\bar{H}(x) \mid \bar{f}(x)$.

Let $\bar{\pi}: \bar{C}\left(\overline{\mathbb{F}}_{q}\right) \rightarrow \mathbb{A}^{1}\left(\overline{\mathbb{F}}_{q}\right)$ be the projection on the $x$-axis. It is clear that $\bar{\pi}$ ramifies at the points $\left(\bar{\theta}_{i}, 0\right) \in \overline{\mathbb{F}}_{q} \times \overline{\mathbb{F}}_{q}$ for $i=0, \ldots, s$ where $\bar{H}\left(\bar{\theta}_{i}\right)=0$. Note that the ordinate of these points is zero, since we assumed that $\bar{H}(x) \mid \bar{f}(x)$. Let $\bar{C}^{\prime}$ be the curve obtained from $\bar{C}$ by removing the ramification points $\left(\bar{\theta}_{i}, 0\right)$ for $i=0, \ldots, s$. Then the coordinate $\operatorname{ring} \bar{A}$ of $\bar{C}^{\prime}$ is

$$
\mathbb{F}_{q}\left[x, y, \bar{H}(x)^{-1}\right] /\left(y^{2}+\bar{h}(x) y-\bar{f}(x)\right)
$$

Let $K$ be a degree $n$ unramified extension of $\mathbb{Q}_{2}$ with valuation $\operatorname{ring} R$ and residue field $R / 2 R=\mathbb{F}_{q}$. Write $\bar{h}(x)=\bar{c} \cdot \prod_{i=0}^{r} \bar{P}_{i}(x)^{t_{i}}$, where the $\bar{P}_{i}(x)$ are monic and irreducible over $\mathbb{F}_{q}$. Let $D=\max _{i} t_{i}$, then $\bar{h}(x)$ divides $\bar{H}(x)^{D}$, since we have the identity $\bar{H}(x)=\prod_{i=0}^{r} \bar{P}_{i}(x)$. Lift $\bar{P}_{i}(x)$ for $i=0, \ldots, r$ to any monic polynomial $P_{i}(x) \in R[x]$. Define $H(x)=\prod_{i=0}^{r} P_{i}(x)$ and $h(x)=c$. $\prod_{i=0}^{r} P_{i}(x)^{t_{i}}$, with $c$ any lift of $\bar{c}$ to $R$. Since $\bar{H}(x)$ divides $\bar{f}(x)$ we can define $\bar{Q}_{\bar{f}}(x)=\bar{f}(x) / \bar{H}(x)$. Let $Q_{f}(x) \in R[x]$ be any monic lift of $\bar{Q}_{\bar{f}}(x)$ and finally set $f(x)=H(x) Q_{f}(x)$. The result is that we have now constructed a lift $C$ of the curve $\bar{C}$ to $R$ defined by the equation

$$
C: y^{2}+h(x) y=f(x) .
$$

Note that due to the careful construction of $C$ we have the following properties: $H(x)|h(x), H(x)| f(x)$ and $h(x) \mid H(x)^{D}$. Let $K^{\text {ur }}$ be the maximal unramified extension of $K$ with valuation ring $R^{\text {ur }}$. For $k=0, \ldots, s$, let $\theta_{k}$ be the zeros of $H(x)$ and note that these are units in $R^{\text {ur }}$. Furthermore, let $\pi: C(\bar{K}) \rightarrow \mathbb{A}^{1}(\bar{K})$ be the projection on the $x$-axis, then the $\left(\theta_{k}, 0\right)$ are ramification points of $\pi$.

Consider the curve $C^{\prime}$ obtained from $C$ by deleting the ramification points $\left(\theta_{k}, 0\right)$ for $k=0, \ldots, s$, then the coordinate $\operatorname{ring} A$ of $C^{\prime}$ is

$$
R\left[x, y, H(x)^{-1}\right] /\left(y^{2}+h(x) y-f(x)\right)
$$


and there exists an involution $\imath$ on $A$ which sends $x$ to $x$ and $y$ to $-y-h(x)$. Let $A^{\dagger}$ denote the weak completion of $A$. Using the equation of the curve, we can represent any element of $A^{\dagger}$ as a series $\sum_{i=-\infty}^{+\infty}\left(U_{i}(x)+V_{i}(x) y\right) S(x)^{i}$, with the degree of $U_{i}(x)$ and $V_{i}(x)$ smaller than the degree of $S(x)$, where $S(x)=H(x)$ if $\operatorname{deg} H>0$ and $S(x)=x$ if $H(x)=1$. The growth condition on the dagger ring implies that there exist real numbers $\delta$ and $\epsilon>0$ such that $\operatorname{ord}_{2}\left(U_{i}(x)\right) \geq \epsilon \cdot|i|+\delta$ and $\operatorname{ord}_{2}\left(V_{i}(x)\right) \geq \epsilon \cdot|i+1|+\delta$, where $\operatorname{ord}_{2}(P(x))$ is defined as $\min _{j} \operatorname{ord}_{2}\left(p_{j}\right)$ for $P(x)=\sum p_{j} x^{j} \in K[x]$.

Lift the 2-power Frobenius $\bar{\sigma}$ on $\mathbb{F}_{q}$ to the Frobenius substitution $\sigma$ on $R$. We extend $\sigma$ to an endomorphism of $A^{\dagger}$ by mapping $x$ to $x^{2}$ and $y$ to $y^{\sigma}$, with

$$
\left(y^{\sigma}\right)^{2}+h(x)^{\sigma} y^{\sigma}-f(x)^{\sigma}=0 \text { and } y^{\sigma} \equiv y^{2} \bmod 2 .
$$

Using Newton iteration we can compute the solution to the above equations as an element of the 2-adic completion $A^{\infty}$ as

$$
W_{k+1} \equiv W_{k}-\frac{W_{k}^{2}+h(x)^{\sigma} W_{k}-f(x)^{\sigma}}{2 W_{k}+h(x)^{\sigma}} \bmod 2^{k+1} .
$$

The only remaining difficulty in the above Newton iteration is that we have to invert $2 W_{k}+h(x)^{\sigma}$ in the ring $A^{\infty}$. Since $h(x) \mid H(x)^{D}$, it makes sense to define $Q_{H}(x):=H(x)^{D} / h(x)$ and we clearly have $1 / h(x)=Q_{H}(x) / H(x)^{D}$. We can now compute the inverse of $2 W_{k}+h(x)^{\sigma}$ as

$$
\frac{Q_{H}(x)^{2}}{H(x)^{2 D} \cdot\left(1+\frac{Q_{H}(x)^{2}\left(2 W_{k}+h(x)^{\sigma}-h(x)^{2}\right)}{H(x)^{2 D}}\right)} .
$$

Note that $h(x)^{\sigma} \equiv h(x)^{2} \bmod 2$, which implies that the denominator in the above formula is invertible in $A^{\infty}$. Contrary to the odd characteristic case it is not immediately clear that the solution $W:=\lim _{k \rightarrow+\infty} W_{k}$ is an element of $A^{\dagger}$. A theorem by Bosch [5] guarantees the existence of such a solution, but does not provide bounds on the rate of convergence. Since these bounds are needed in the complexity analysis, we prove the following lemma.

Lemma 1 For $k \geq 1$, let $W_{k}=\sum_{i=-L_{k}}^{A_{k}} U_{i}(x) S(x)^{i}+\sum_{i=-L_{k}}^{B_{k}} V_{i}(x) S(x)^{i} y \in A$, with $S(x)=H(x)$ if $\operatorname{deg} H>0, S(x)=x$ if $H(x)=1$ and $\operatorname{deg} U_{i}<\operatorname{deg} S$, $\operatorname{deg} V_{i}<\operatorname{deg} S$ satisfy

$$
W_{k}^{2}+h(x)^{\sigma} W_{k}-f(x)^{\sigma} \equiv 0 \bmod 2^{k} \quad \text { and } \quad W_{k} \equiv y^{2} \bmod 2
$$

with $U_{A_{k}} \neq 0, V_{B_{k}} \neq 0, U_{-L_{k}} \neq 0$ or $V_{-L_{k}} \neq 0$ and such that $U_{i}=0$ or $\operatorname{ord}_{2}\left(U_{i}(x)\right)<k$ for $-L_{k} \leq i \leq A_{k}$ and $V_{i}=0$ or $\operatorname{ord}_{2}\left(V_{i}(x)\right)<k$ for $-L_{k} \leq$ $i \leq B_{k}$. Then $A_{k}, B_{k}$ and $L_{k}$ can be bounded for $k \geq 2$ as

$$
\begin{aligned}
& A_{k} \leq 2(k-1)\left(d_{S}^{f}-2 d_{S}^{h}\right)+2 d_{S}^{h}, \\
& B_{k} \leq 2(k-2)\left(d_{S}^{f}-2 d_{S}^{h}\right)+\left(d_{S}^{f}-d_{S}^{h}\right), \\
& L_{k} \leq 4(k-1) D-2 D,
\end{aligned}
$$

with $d_{S}^{f}:=\operatorname{deg} f / \operatorname{deg} S$ and $d_{S}^{h}:=\operatorname{deg} h / \operatorname{deg} S$. 
Proof: An easy calculation shows that $W_{1} \equiv f(x)-h(x) y \bmod 2$, thus $A_{1} \leq d_{S}^{f}$, $B_{1} \leq d_{S}^{h}, L_{1} \leq 0$ and that

$$
W_{2} \equiv \frac{f(x)^{\sigma}-f(x)^{2}-h(x)^{\sigma} f(x)}{h(x)^{2}}+y \frac{h(x)^{\sigma}+2 f(x)}{h(x)} \bmod 4
$$

which implies that $W_{2}$ satisfies the lemma. The Newton iteration (1) can be rewritten as

$$
h(x)^{2} W_{k+1} \equiv-W_{k}^{2}+\left(h(x)^{2}-h(x)^{\sigma}\right) W_{k}+f(x)^{\sigma} \bmod 2^{k+1} .
$$

Let $\alpha_{k}(x):=\sum_{i=-L_{k}}^{A_{k}} U_{i}(x) S(x)^{i}$ and $\beta_{k}(x):=\sum_{l=-L_{k}}^{B_{k}} V_{i}(x) S(x)^{i}$ such that $W_{k}=\alpha_{k}(x)+\beta_{k}(x) y$. Note that $W_{k} \equiv W_{k-1} \bmod 2^{k-1}$, so we can define

$$
\Delta_{\alpha, k}(x):=\frac{\alpha_{k}(x)-\alpha_{k-1}(x)}{2^{k-1}} \quad \text { and } \quad \Delta_{\beta, k}(x):=\frac{\beta_{k}(x)-\beta_{k-1}(x)}{2^{k-1}},
$$

for $k \geq 1$ and $\Delta_{\alpha, 0}(x):=\Delta_{\beta, 0}(x):=0$. It is clear that $W_{k}$ can be written as

$$
W_{k}=\Delta_{\alpha, 1}+2 \Delta_{\alpha, 2}+\cdots+2^{k-1} \Delta_{\alpha, k}+y\left(\Delta_{\beta, 1}+2 \Delta_{\beta, 2}+\cdots+2^{k-1} \Delta_{\beta, k}\right) .
$$

Plugging this into the Newton iteration gives the following equation

$$
\begin{aligned}
& h(x)^{2} W_{k+1} \equiv-\sum_{\substack{1 \leq i<j \\
i+j-1<k+1}} 2^{i+j-1}\left(\Delta_{\alpha, i} \Delta_{\alpha, j}+(f(x)-h(x) y) \Delta_{\beta, i} \Delta_{\beta, j}\right) \\
& -y \sum_{i+j-1<k+1} 2^{i+j-1} \Delta_{\alpha, i} \Delta_{\beta, j}-\sum_{2(i-1)<k+1} 2^{2(i-1)}\left(\Delta_{\alpha, i}^{2}+(f(x)-h(x) y) \Delta_{\beta, i}^{2}\right) \\
& +\left(h(x)^{2}-h(x)^{\sigma}\right) \sum_{i<k+1} 2^{i-1}\left(\Delta_{\alpha, i}+\Delta_{\beta, i} y\right)+f(x)^{\sigma} \bmod 2^{k+1} .
\end{aligned}
$$

By definition $Q_{H}(x) h(x)=H(x)^{D}$, which implies $1 / h(x)^{2}=Q_{H}(x)^{2} / H(x)^{2 D}$ and $\operatorname{deg} Q_{H}=D \operatorname{deg} H-\operatorname{deg} h$. Since $\operatorname{deg} \Delta_{\alpha, i} \leq A_{i}$ and $\operatorname{deg} \Delta_{\beta, i} \leq B_{i}$, we conclude that $A_{k+1}$ is less than or equal to

$$
\begin{aligned}
\max \left(\max _{i+j<k+2}\left(A_{i}+A_{j}, B_{i}+B_{j}+d_{S}^{f}\right),\right. & \max _{2 i<k+3}\left(2 A_{i}, 2 B_{i}+d_{S}^{f}\right), \\
& \left.\max _{i<k+1} A_{i}+2 d_{S}^{h}, 2 d_{S}^{f}\right)-2 d_{S}^{h} .
\end{aligned}
$$

Using the bounds given in (3) for $A_{i}$ and $B_{i}$ and the bounds $A_{1} \leq d_{S}^{f}, B_{1} \leq d_{S}^{h}$ and $L_{1} \leq 0$, we see that $A_{k+1}$ also satisfies the bounds (3). A similar reasoning can be used to prove that $B_{k+1}$ and $L_{k+1}$ also satisfy the given bounds.

Lemma 1 implies that the $q$-power Frobenius $\bar{F}$ can be lifted to an endomorphism $F$ on the dagger ring $A^{\dagger}$, since we can simply take $F:=\sigma^{n}$. If we are to compute the action of $F$ on the first Monsky-Washnitzer cohomology group 
$H^{1}(\bar{A} / K)$, we need to determine a basis for $H^{1}(\bar{A} / K)$. Following Kedlaya, we proceed in two steps: we first determine a basis for the algebraic de Rham cohomology group $H_{D R}^{1}(A / K)$ and then show that this is also a basis for $H^{1}(\bar{A} / K)$.

Analogous to the odd characteristic case, the algebraic de Rham cohomology $H_{D R}^{1}(A / K)$ of $A$ splits into eigenspaces under the hyperelliptic involution. The positive eigenspace $H_{D R}^{1}(A / K)^{+}$is generated by $x^{i} / H(x) d x$ for $i=0, \ldots, s$ and the negative eigenspace $H_{D R}^{1}(A / K)^{-}$is generated by $x^{i} y d x$ for $i=0, \ldots, 2 g-1$. Note that the positive eigenspace corresponds to the deleted ramification points $\left(\theta_{k}, 0\right)$ for $k=0, \ldots, s$. Every element of $H_{D R}^{1}(A / K)$ can be written as a linear combination of differentials of the form $x^{k} H(x)^{m} y^{l} d x, x^{k} H(x)^{m} y^{l} d y$ with $k, l \in \mathbb{N}$ and $m \in \mathbb{Z}$. Using the equation of the curve, we can reduce to the case $l=0$ or 1 . Since $d\left(x^{k} H(x)^{m} y\right)$ and $d\left(x^{k} H(x)^{m} y^{2}\right)$ are exact, we conclude that $H_{D R}^{1}(A / K)$ is generated by differentials of the form $x^{k} H(x)^{m} d x$ and $x^{k} H(x)^{m} y d x$ with $k \in \mathbb{N}$ and $m \in \mathbb{Z}$.

It is clear that $x^{k} H(x)^{m} d x$ is exact for $k \in \mathbb{N}$ and $m \geq 0$. If $\operatorname{deg} H>0$ and $m<0$ we can assume that $0 \leq k<\operatorname{deg} H$ and since $H(x)$ is squarefree we can write $x^{k}$ as $A(x) H(x)+B(x) H^{\prime}(x)$, which leads to

$$
x^{k} H(x)^{m} d x=A(x) H(x)^{m+1} d x+B(x) H^{\prime}(x) H(x)^{m} d x .
$$

Since $d\left(B(x) H(x)^{m+1}\right)$ is exact we can reduce the above differential further for $m<-1$ by using the relation

$$
B(x) H^{\prime}(x) H(x)^{m} d x \equiv-\frac{B^{\prime}(x) H(x)^{m+1}}{m+1} d x,
$$

where $\equiv$ means equality modulo exact differentials. As a result we can now reduce any form $x^{k} H(x)^{m} d x$ to a linear combination of the differentials $x^{i} / H(x) d x$ for $i=0, \ldots, s$.

For $m>0$ we can reduce the differential form $x^{k} H(x)^{m} y d x$ for $k \in \mathbb{N}$ if we know how to reduce the form $x^{i} y d x$ for $i \in \mathbb{N}$. Rewriting the equation of the curve as $(2 y+h(x))^{2}=4 f(x)+h(x)^{2}$ and differentiating both sides leads to $(2 y+h(x)) d(2 y+h(x))=\left(2 f^{\prime}(x)+h(x) h^{\prime}(x)\right) d x$. Furthermore, for all $j \geq 1$, we have the following relations

$$
\begin{aligned}
x^{j}\left(2 f^{\prime}(x)+h(x) h^{\prime}(x)\right)(2 y+h(x)) d x & =x^{j}(2 y+h(x))^{2} d(2 y+h(x)) \\
& \equiv-\frac{1}{3}(2 y+h(x))^{3} d x^{j} \\
& =-\frac{j}{3} x^{j-1}\left(4 f(x)+h(x)^{2}\right)(2 y+h(x)) d x .
\end{aligned}
$$

Since $P(x) h(x) d x$ is exact for any polynomial $P(x) \in K[x]$, we finally obtain that

$$
\left[x^{j}\left(2 f^{\prime}(x)+h(x) h^{\prime}(x)\right)+\frac{j}{3} x^{j-1}\left(4 f(x)+h(x)^{2}\right)\right] y d x \equiv 0 .
$$

The polynomial between brackets has degree $2 g+j$ and its leading coefficient is $2(2 g+1)+4 j / 3 \neq 0$. Note that the formula is also valid for $j=0$. This means 
that we can reduce $x^{i} y d x$ for any $i \geq 2 g$ by subtracting a suitable multiple of the above differential for $j=i-2 g$.

For $m<0$ we need an extra trick to reduce the form $x^{k} H(x)^{m} y d x$ with $k \in \mathbb{N}$. Recall that $Q_{f}(x)=f(x) / H(x)$ and since the curve is non-singular, we conclude that $\operatorname{gcd}\left(Q_{f}(x), H(x)\right)=1$. Furthermore, $H(x)$ has no repeated roots which implies $\operatorname{gcd}\left(H(x), Q_{f}(x) H^{\prime}(x)\right)=1$. Let $i=-m>0$, then we can partially reduce $x^{k} y / H(x)^{i} d x$ by writing $x^{k}$ as $A(x) H(x)+B(x) Q_{f}(x) H^{\prime}(x)$, which leads to

$$
\frac{x^{k}}{H(x)^{i}} y d x=\frac{A(x)}{H(x)^{i-1}} y d x+\frac{B(x) Q_{f}(x) H^{\prime}(x)}{H(x)^{i}} y d x .
$$

The latter differential form can be reduced using the following congruence

$$
\begin{aligned}
\frac{B(x)}{H(x)^{i}}\left(2 f^{\prime}(x)+h(x) h^{\prime}(x)\right)(2 y+ & h(x)) d x \\
& =\frac{B(x)}{H(x)^{i}}(2 y+h(x))^{2} d(2 y+h(x)) \\
& \equiv-\frac{1}{3}(2 y+h(x))^{3} d\left(\frac{B(x)}{H(x)^{i}}\right) .
\end{aligned}
$$

Substituting the expressions $h(x)=Q_{h}(x) H(x), f(x)=Q_{f}(x) H(x)$ and $(2 y+h(x))^{2}=4 f(x)+h(x)^{2}$, we get

$$
\begin{aligned}
& \frac{B(x) Q_{f}(x) H^{\prime}(x)}{H(x)^{i}} y d x \equiv \\
& \quad \frac{B\left(i H^{\prime} Q_{h}^{2}-6 Q_{f}^{\prime}-3 Q_{h} h^{\prime}\right)-B^{\prime}\left(4 Q_{f}+Q_{h} h\right)}{(6-4 i) H^{i-1}} y d x+\frac{I}{H} d x,
\end{aligned}
$$

where $I(x) / H(x) d x$ is a suitable invariant differential. As a result we can write any form $x^{k} H(x)^{m} y d x$ for $k \in \mathbb{N}$ and $m \in \mathbb{Z}$ as a linear combination of the differentials $x^{i} y d x$ for $i=0, \ldots, 2 g-1$ and $x^{i} / H(x) d x$ for $i=0, \ldots, s$.

To show that the Monsky-Washnitzer cohomology $H^{1}(\bar{A} / K)$ is generated by the same differential forms as the algebraic de Rham cohomology, we need to bound the denominators introduced during the reduction process.

Lemma 2 Let $A:=R[x, y] /\left(y^{2}+h(x) y-f(x)\right)$ and suppose that

$$
x^{r} y d x=\sum_{i=0}^{2 g-1} a_{i} x^{i} y d x+d S
$$

with $r \in \mathbb{N}, a_{i} \in K$ and $S \in A \otimes K$. Then $2^{m} a_{i} \in R, 2^{m^{\prime}} S-\beta \in A$, where $m=3+\left\lfloor\log _{2}(r+g+1)\right\rfloor, m^{\prime}=1+m+\left\lfloor\log _{2}(2 g+\operatorname{deg} h)\right\rfloor$ and $\beta$ some suitable element in $K$. 
Proof: The proof has two distinct parts. The first part is similar to Kedlaya's argument in [17, Lemma 3], and is based on a local analysis around the point at infinity of the curve $C$. Put $t=x^{g} / y$, then one easily verifies that

$$
x=t^{-2}\left(1+\sum_{j=1}^{\infty} \alpha_{j} t^{j}\right) \quad \text { and } \quad y=t^{-2 g-1}\left(1+\sum_{j=1}^{\infty} \beta_{j} t^{j}\right),
$$

with $\alpha_{j}, \beta_{j} \in R$. To see this, put $z=1 / x$, rewrite the equation of the curve $C$ as $z+t z^{g+1} h(1 / z)-t^{2} z^{2 g+1} f(1 / z)=0$ and write $z$ as a power series in $t$ using Newton iteration. The relation (4) can be rewritten as

$$
2^{m-1} x^{r}(2 y+h(x)) d x=\sum_{i=0}^{2 g-1} 2^{m-1} a_{i} x^{i}(2 y+h(x)) d x+d T,
$$

with $T \in A \otimes K$. Considering the involution $\imath$ of $A$ which sends $x$ to $x$ and $2 y+h(x)$ to $-(2 y+h(x))$, we see that we can write $T=\sum_{i=0}^{N} A_{i} x^{i}(2 y+h(x))$, with $N$ big enough and $A_{i} \in K$. This yields

$$
\begin{aligned}
& 2^{m-1} x^{r}(2 y+h(x)) d x-\sum_{i=0}^{2 g-1} 2^{m-1} a_{i} x^{i}(2 y+h(x)) d x \\
&=d\left(\sum_{i=0}^{N} A_{i} x^{i}(2 y+h(x))\right) .
\end{aligned}
$$

In the above equation we express $x$ and $y$ in terms of $t$ using equalities (5). Since $x^{i} y=t^{-2 i-2 g-1}+\cdots$, we get $x^{i}(2 y+h(x)) d x=\left(-4 t^{-2 i-2 g-4}+\cdots\right) d t$, which yields

$$
\begin{aligned}
& \underset{j=-\max (2 r+2 g+4,6 g+2)}{2^{m-1} \gamma_{j} t^{j}} d t \\
& \quad=d\left(\sum_{i=0}^{N} 2 A_{i}\left(t^{-2 i-2 g-1}+\cdots\right)+A_{i}\left(c t^{-2 i-2 \operatorname{deg} h}+\cdots\right)\right),
\end{aligned}
$$

with $\gamma_{j} \in K$ for all $j$ and $\gamma_{j} \in R$ when $j<-2(2 g-1)-2 g-4=-6 g-2$ and $c$ the leading coefficient of $h(x)$. Note that $c$ is a unit in $R$. Integrating with respect to $t$ and dividing by 2 gives

$$
\sum_{j \geq-\max (2 r+2 g+3,6 g+1)} \gamma_{j^{j}}^{j}=\sum_{i=0}^{N} A_{i}\left(t^{-2 i-2 g-1}+\cdots\right)+\sum_{i=0}^{N} \frac{A_{i}}{2}\left(c t^{-2 i-2 \operatorname{deg} h}+\cdots\right),
$$

with $\gamma_{j}^{\prime} \in K$ for all $j$ and $\gamma_{j}^{\prime} \in R$ when $j<-6 g-1$. Indeed the integration process introduces denominators which become integral after multiplication with $2^{\lfloor\log (2 r+2 g+2)\rfloor}=2^{m-2}$ if $r \geq 2 g-1$. A first consequence of $(7)$ is that $A_{i}=0$ for all $i>\max (r+1,2 g)$. We claim that (7) implies that $A_{i} \in R$ for all $i>2 g$. 
Suppose the claim is false. Then let $i_{0}$ be the largest integer with $i_{0}>2 g$ and $A_{i_{0}} \notin R$. Note that $-2 i_{0}-2 g-1<-6 g-1$, since $i_{0}>2 g$. Hence the monomials in the left hand side of (7) with degree $\leq-2 i_{0}-2 g-1$ have coefficients in $R$. Moreover the monomials of degree $<-2 i_{0}-2 g-1$, in the first sum in the right hand side of (7) also have coefficients in $R$, but this is false for the monomial of degree $-2 i_{0}-2 g-1$. Hence the second sum in the right hand side of (7) contains a monomial of degree $-2 i_{0}-2 g-1$ whose coefficient is not in $R$. That means that there is a maximal $i_{1}$ with $A_{i_{1}} / 2 \notin R$ and $-2 i_{1}-2 \operatorname{deg} h \leq-2 i_{0}-2 g-1$. Because of parity we have that $-2 i_{1}-2 \operatorname{deg} h<-2 i_{0}-2 g-1$. Since $c$ is a unit, the right hand side of (7) contains a monomial of degree $-2 i_{1}-2 \operatorname{deg} h<-2 i_{0}-2 g-1$ whose coefficient is not in $R$. But this contradicts what we said about the left hand side. This finishes the claim that $A_{i} \in R$ for all $i>2 g$.

We now turn to the second part of the proof. Note that $(2 y+h(x))^{2}=v(x)$ with $v(x):=4 f(x)+h(x)^{2}$. Moreover, $d(2 y+h(x))=\frac{w(x)}{2 y+h(x)} d x$, where $w(x):=$ $2 f^{\prime}(x)+h(x) h^{\prime}(x)$. We will use these formulae to deduce from (6) a relation which does not involve $y$. For this purpose we multiply (6) with $\frac{2 y+h(x)}{d x}=\frac{w(x)}{d(2 y+h(x))}$ obtaining

$$
2^{m-1} x^{r} v(x)-\sum_{i=0}^{2 g-1} 2^{m-1} a_{i} x^{i} v(x)=\sum_{i=0}^{N} A_{i} i x^{i-1} v(x)+\sum_{i=0}^{N} A_{i} x^{i} w(x) .
$$

We rewrite this in the form

$$
\left(\sum_{i=0}^{2 g-1} 2^{m-1} a_{i} x^{i}\right) v(x)+\left(\sum_{i=0}^{2 g} A_{i} i x^{i-1}\right) v(x)+\left(\sum_{i=0}^{2 g} A_{i} x^{i}\right) w(x)=F(x),
$$

where

$$
F(x):=2^{m-1} x^{r} v(x)-\sum_{i=2 g+1}^{N} A_{i} i x^{i-1} v(x)-\sum_{i=2 g+1}^{N} A_{i} x^{i} w(x)
$$

is a polynomial over $R$, since $A_{i} \in R$ for all $i>2 g$. From equations (8) and (9) it follows that $\sum_{i=0}^{2 g} A_{i} \theta_{k}^{i}$ has valuation $\geq 0$ for each root $\theta_{k}$ of $H(x)$, because $v\left(\theta_{k}\right)=0$ and $w\left(\theta_{k}\right) \neq 0$. To get rid of the disturbing factor 2 in the definition of $w(x)$, we consider $q(x):=h^{\prime}(x) H(x) / h(x) \in R[x]$ and $u(x):=\frac{1}{2}(w(x)-$ $q(x) v(x) / H(x))=f^{\prime}(x)-2 q(x) f(x) / H(x)$. Note that $u(x) \in R[x], \operatorname{deg} q=$ $\max (0, \operatorname{deg} H-1), \operatorname{deg} u=2 g$ and that the leading coefficient of $u(x)$ is a unit in $R$. Rewrite equation (8) in such a way that $w(x)$ gets replaced by $u(x)$ :

$$
\begin{aligned}
\left(\sum_{i=0}^{2 g-1} 2^{m-1} a_{i} x^{i}+\sum_{i=0}^{2 g} A_{i} i x^{i-1}+\frac{q(x)}{H(x)}\right. & \left.\sum_{i=0}^{2 g} A_{i} x^{i}\right) v(x) \\
& +\left(\sum_{i=0}^{2 g} 2 A_{i} x^{i}\right) u(x)=F(x) .
\end{aligned}
$$


Write $q(x) \sum_{i=0}^{2 g} A_{i} x^{i}=H(x) \sum_{i=0}^{2 g-1} B_{i} x^{i}+\operatorname{Rem}(x)$, with $\operatorname{Rem}(x) \in K[x]$ of degree $<\operatorname{deg} H$. Since $\sum_{i=0}^{2 g} A_{i} \theta_{k}^{i}$ has valuation $\geq 0$ for each root $\theta_{k}$ of $H(x)$, the same holds for $\operatorname{Rem}\left(\theta_{k}\right)$. Thus $\operatorname{Rem}(x) \in R[x]$ since the discriminant of $H(x)$ is a unit in $R$. Hence

$$
\begin{aligned}
\left(\sum_{i=0}^{2 g-1}\left(2^{m-1} a_{i}+(i+1) A_{i+1}+B_{i}\right) x^{i}\right) v(x)+ & \left(\sum_{i=0}^{2 g} 2 A_{i} x^{i}\right) u(x) \\
& =F(x)-\frac{\operatorname{Rem}(x) v(x)}{H(x)} .
\end{aligned}
$$

We consider (10) as a system of $4 g+1$ linear equations in the unknowns $2^{m-1} a_{i}+$ $(i+1) A_{i+1}+B_{i}$ for $i=0, \ldots, 2 g-1$ and $2 A_{i}$ for $i=0, \ldots, 2 g$. The determinant of this system is the resultant $\operatorname{Res}(v(x), u(x))$ of $v(x)$ and $u(x)$ because $\operatorname{deg} v=2 g+1$ and $\operatorname{deg} u=2 g$. This resultant is a unit in $R$ because the valuation of $v(\xi)$ is zero for each root $\xi$ of $u(x)$, since the resultant of $f^{\prime}(x)$ and $h(x)$ is a unit. We conclude that the solutions of the linear system are elements of $R$, thus $2 A_{i} \in R$ and $2^{m-1} a_{i}+(i+1) A_{i+1}+B_{i} \in R$. From the definition of the $B_{i}$ it follows that $2 B_{i} \in R$ since $2 A_{i} \in R$ and $\operatorname{Rem}(x) \in R[x]$. Hence $2^{m} a_{i} \in R$, which concludes the proof of Lemma 2 .

Remark Lemma 2 remains valid when we replace $\sum_{i=0}^{2 g-1}$ by $\sum_{i=\kappa}^{2 g-1+\kappa}$ whenever $r \geq \kappa \in \mathbb{N}$. The proof is the same, except that we also have to show that $A_{i}=0$ for all $i<\kappa$. This follows from (6) using a local analysis at a point on the curve with $x=\theta_{k}$. Such a local analysis is given in the proof of Lemma 3 below.

Lemma 3 Let $A:=R\left[x, y, H(x)^{-1}\right] /\left(y^{2}+h(x) y-f(x)\right)$ with $\operatorname{deg} h>0$ and suppose that

$$
\frac{B(x)}{H(x)^{r}} y d x=\sum_{i=0}^{2 g-1} a_{i} x^{i} y d x+\sum_{i=0}^{s} \frac{b_{i} x^{i}}{H(x)} d x+d S,
$$

where $r \in \mathbb{N}, B(x) \in R[x]$ of degree $<\operatorname{deg} H, a_{i}, b_{i} \in K$ and $S \in A \otimes K$. Then $2^{m} a_{i} \in R, 2^{m^{\prime}} b_{i} \in R, 2^{m^{\prime}} S-\beta \in A$, with $m=3+\left\lfloor\log _{2}(r+1)\right\rfloor, m^{\prime}=$ $1+m+\left\lfloor\log _{2}(2 g+\operatorname{deg} h)\right\rfloor$ and $\beta$ some suitable element in $K$.

Proof: The proof again consists of two distinct parts. The first part is similar to Kedlaya's argument in [17, Lemma 2] and is based on a local analysis around the ramification points $\left(\theta_{k}, 0\right)$ for $k=0, \ldots, s$. In the completion of the local ring of the curve at $\left(\theta_{k}, 0\right)$ we can write

$$
x-\theta_{k}=\gamma_{k, 2} y^{2}+\sum_{j \geq 3} \gamma_{k, j} y^{j},
$$

with $\gamma_{k, j} \in R^{\mathrm{ur}}$ and $\gamma_{k, 2}$ a unit in $R^{\mathrm{ur}}$. Indeed, to see this write $h(x)$ and $f(x)$ as a Taylor expansion around $\theta_{k}$ and use the equation of the curve and the condition $f^{\prime}\left(\theta_{k}\right) \not \equiv 0 \bmod 2$, to express $x-\theta_{k}$ as a power series in $y$ using Newton iteration. 
Applying the involution $\imath$ to equation (11), we see that this relation implies

$$
\begin{aligned}
2^{m-1} B(x) H(x)^{-r}(2 y+h(x)) d x & -\sum_{i=0}^{2 g-1} 2^{m-1} a_{i} x^{i}(2 y+h(x)) d x \\
& =d\left(\sum_{i=-N}^{M} B_{i}(x) H(x)^{i}(2 y+h(x))\right),
\end{aligned}
$$

with $N$ and $M$ large enough integers. Expressing $x-\theta_{k}$ in terms of $y$, we get $B_{i}(x) H(x)^{i}=u_{k, i} B_{i}\left(\theta_{k}\right) y^{2 i}+\cdots$ with $u_{k, i}$ a unit in $R^{\text {ur }}$. Substituting this in equation (12) and dividing by 2 we obtain

$$
\begin{aligned}
& 2^{m-2} \sum_{j \geq-2 r+2} \gamma_{k, j}^{\prime} y^{j} d y \\
& =d\left(\sum_{i=-N}^{M} u_{k, i} B_{i}\left(\theta_{k}\right) y^{2 i+1}+\frac{u_{k, i} B_{i}\left(\theta_{k}\right)}{2} \frac{\gamma_{k, 2}^{m_{k}} h^{\left(m_{k}\right)}\left(\theta_{k}\right)}{m_{k} !} y^{2 i+2 m_{k}}+\cdots\right)
\end{aligned}
$$

with $\gamma_{k, j}^{\prime} \in K^{\mathrm{ur}}$ for all $j$ and $\gamma_{k, j}^{\prime} \in R^{\mathrm{ur}}$ when $j \leq 1$. Integrating the left hand side of this equation with respect to $y$ yields a series whose terms of degree $\leq 2$ have coefficients in $R^{\mathrm{ur}}$. The leading term of the right hand side is $u_{k,-N} B_{-N}\left(\theta_{k}\right) y^{-2 N+1}$, which implies that $B_{-N}\left(\theta_{k}\right)$ is integral for $k=0, \ldots, s$. Since the discriminant of $H(x)$ is a unit in $R$ we conclude that $B_{-N}(x)$ has integral coefficients. Bringing the integral terms to the left hand side and repeating the same argument, shows that $B_{i}(x) \in R[x]$ for $i=-N, \ldots, 0$. This terminates the first part of the proof.

The second part of the proof proceeds along the same lines as in Lemma 2. Rewrite the sum $\sum_{i=1}^{M} B_{i}(x) H(x)^{i}(2 y+h(x))$ as $\sum_{i=0}^{M^{\prime}} A_{i} x^{i}(2 y+h(x))$ with $M^{\prime} \in \mathbb{N}$ and $A_{i} \in K$. Using the same formulae as in Lemma 2 we deduce from (12) a relation which does not involve $y$ by multiplying both sides with $\frac{2 y+h(x)}{d x}=\frac{w(x)}{d(2 y+h(x))}$, which leads to

$$
\begin{aligned}
& 2^{m-1} \frac{B(x)}{H(x)^{r}} v(x)-\sum_{i=0}^{2 g-1} 2^{m-1} a_{i} x^{i} v(x)=\sum_{i=-N}^{0} B_{i}(x) H(x)^{i} w(x)+\sum_{i=0}^{M^{\prime}} A_{i} x^{i} w(x) \\
&+\sum_{i=-N}^{0}\left(B_{i}(x) i H(x)^{i-1} H^{\prime}(x)+B_{i}^{\prime}(x) H(x)^{i}\right) v(x)+\sum_{i=0}^{M^{\prime}} A_{i} i x^{i-1} v(x) .
\end{aligned}
$$

Comparing the valuation at infinity of both sides shows that $A_{i}=0$ for $i>2 g$. We can therefore rewrite the above equation in the form

$$
\left(\sum_{i=0}^{2 g-1} 2^{m-1} a_{i} x^{i}\right) v(x)+\left(\sum_{i=0}^{2 g} A_{i} i x^{i-1}\right) v(x)+\left(\sum_{i=0}^{2 g} A_{i} x^{i}\right) w(x)=F(x)
$$


where

$$
\begin{aligned}
F(x):=2^{m-1} \frac{B(x)}{H(x)^{r}} v(x) & -\sum_{i=-N}^{0} B_{i}(x) H(x)^{i} w(x) \\
& -\sum_{i=-N}^{0}\left(B_{i}(x) i H(x)^{i-1} H^{\prime}(x)+B_{i}^{\prime}(x) H(x)^{i}\right) v(x)
\end{aligned}
$$

is a polynomial over $R$ since the $B_{i}(x) \in R[x]$ for $i=-N, \ldots, 0$ and the lefthand side of (13) is a polynomial. From the definition of the $A_{i}$ it follows that $H(x)$ divides $\sum_{i=0}^{2 g} A_{i} x^{i}$. It is now easy to see that the rest of the proof is exactly the same as in the proof of Lemma 2 with $\operatorname{Rem}(x)=0$, hence $2^{m} a_{i} \in R$ and this concludes the proof of Lemma 3.

Remark Lemma 3 remains valid when we replace the term $\sum_{i=0}^{2 g-1} a_{i} x^{i} y d x$ in equation (11) by $\sum_{i=-\kappa}^{\Delta-\kappa} C_{i}(x) H(x)^{i} y d x$, with $\Delta=\lfloor(2 g-1) / \operatorname{deg} H\rfloor$ and $C_{i}(x) \in K[x]$ of degree $<\operatorname{deg} H$ whenever $r \geq \kappa \in \mathbb{N}$. The proof is exactly the same.

Remark If $r=0$, then in the above proof the $B_{i}(x)$ are zero for all $i \leq 0$, and for $0 \leq i \leq 2 g-1$ the $a_{i}$ are completely determined by (13) as we saw by considering resultants. This shows that the $x^{i} y d x$ for $i=0, \ldots, 2 g-1$ and the $\frac{x^{i}}{H(x)} d x$ for $i=0, \ldots, s$ are linearly independent in $H_{D R}^{1}(A / K)$.

An immediate consequence of Lemmata 2 and 3 is that the basis for $H_{D R}^{1}(A / K)$ also generates $H^{1}(\bar{A} / K)$ : reducing a differential $\sum_{k, l} a_{k, l} x^{k} S(x)^{l} y d x \in D^{1}\left(A^{\dagger}\right)$ with $k, l \in \mathbb{Z}$ and $0 \leq k<\operatorname{deg} S$ introduces denominators whose valuation grows logarithmically in $|l|$, whereas the valuation of $a_{k, l}$ grows linearly in $|l|$. Combining this with the above remark, we conclude that the basis for $H_{D R}^{1}(A / K)$ is also a basis for $H^{1}(\bar{A} / K)$.

Under the action of the hyperelliptic involution, the Monsky-Washnitzer cohomology $H^{1}(\bar{A} / K)$ decomposes as the direct sum of the $\imath$-invariant part $H^{1}(\bar{A} / K)^{+}$and the $\imath$-anti-invariant part $H^{1}(\bar{A} / K)^{-}$. Let $r_{k}$ be the number of ramification points $(\bar{\theta}, 0)$ defined over $\mathbb{F}_{q^{k}}$, then the Lefschetz fixed point formula applied to $C^{\prime}$ gives

$$
\begin{aligned}
\# C\left(\mathbb{F}_{q^{k}}\right)-r_{k} & =\# C^{\prime}\left(\mathbb{F}_{q^{k}}\right) \\
& =\operatorname{Tr}\left(q^{k} F_{*}^{-k} \mid H^{0}(\bar{A} / K)\right)-\operatorname{Tr}\left(q^{k} F_{*}^{-k} \mid H^{1}(\bar{A} / K)\right) \\
& =q^{k}-\operatorname{Tr}\left(q^{k} F_{*}^{-k} \mid H^{1}(\bar{A} / K)^{+}\right)-\operatorname{Tr}\left(q^{k} F_{*}^{-k} \mid H^{1}(\bar{A} / K)^{-}\right) \\
& =q^{k}-r_{k}-\operatorname{Tr}\left(q^{k} F_{*}^{-k} \mid H^{1}(\bar{A} / K)^{-}\right) .
\end{aligned}
$$

Let $\widetilde{C}$ be the unique smooth projective curve birational to $\bar{C}$, then

$$
\# \widetilde{C}\left(\mathbb{F}_{q^{k}}\right)=q^{k}+1-\operatorname{Tr}\left(q^{k} F_{*}^{-k} \mid H^{1}(\bar{A} / K)^{-}\right)=q^{k}+1-\sum_{i=1}^{2 g} \alpha_{i}^{k},
$$


with $\alpha_{i}$ the eigenvalues of $q F_{*}^{-1}$ on $H^{1}(\bar{A} / K)^{-}$. The Weil conjectures imply that there exist $2 g$ algebraic integers $\beta_{1}, \ldots, \beta_{2 g}$ with $\beta_{i} \beta_{g+i}=q$ for $i=1, \ldots, g$ and $\left|\beta_{i}\right|=\sqrt{q}$ for $i=1, \ldots, 2 g$, such that for all $k>0$ we have $\# \widetilde{C}\left(\mathbb{F}_{q^{k}}\right)=$ $q^{k}+1-\sum_{i=1}^{2 g} \beta_{i}^{k}$. Comparing both expressions, we see that we can relabel the $\alpha_{i}$ such that $\alpha_{i}=\beta_{i}$ for $i=1, \ldots, 2 g$. Since then $\alpha_{i} \alpha_{g+i}=q$, the $\alpha_{i}$ are also the eigenvalues of $F_{*}$ on $H^{1}(\bar{A} / K)^{-}$. Let $\chi(t)$ be the characteristic polynomial of $F_{*}$ on $H^{1}(\bar{A} / K)^{-}$, then we can finally recover the zeta function $Z\left(\widetilde{C} / \mathbb{F}_{q} ; t\right)$ as

$$
Z\left(\widetilde{C} / \mathbb{F}_{q} ; t\right)=\frac{t^{2 g} \chi(1 / t)}{(1-t)(1-q t)} .
$$

\section{Algorithm and Complexity}

Using the formulae of the previous section, we describe an algorithm to compute the characteristic polynomial of Frobenius $\chi(t)$ and the zeta function of a smooth projective hyperelliptic curve $\widetilde{C}$ of genus $g$ over $\mathbb{F}_{q}$ with $q=2^{n}$.

\subsection{Precision of Computation}

We have shown that $\chi(t)=t^{2 g}+a_{1} t^{2 g-1}+\cdots+a_{2 g}$ can be computed as the characteristic polynomial of $F_{*}$ on $H^{1}(\bar{A} / K)^{-}$. The Weil conjectures imply that $q^{g-i} a_{i}=a_{2 g-i}$, so it suffices to compute $a_{1}, \ldots, a_{g}$. Furthermore, for $i=1, \ldots, g$ the $a_{i}$ can be bounded by

$$
\left|a_{i}\right| \leq\left(\begin{array}{c}
2 g \\
i
\end{array}\right) q^{i / 2} \leq\left(\begin{array}{c}
2 g \\
g
\end{array}\right) q^{g / 2} \leq 2^{2 g} q^{g / 2} .
$$

Therefore it suffices to compute the action of $F_{*}$ on a basis of $H^{1}(\bar{A} / K)^{-}$modulo $2^{B}$ with

$$
B \geq\left\lceil\log _{2}\left(2\left(\begin{array}{c}
2 g \\
g
\end{array}\right) q^{g / 2}\right)\right\rceil .
$$

However, it is not sufficient to compute $y^{\sigma}$ modulo $2^{B}$ since we need to take into account the loss of precision introduced during the reduction process of the differential forms. Let $y^{\sigma} \equiv \alpha_{N}+\beta_{N} y \bmod 2^{N}$ and write $\beta_{N}=\sum_{i=-L_{N}}^{B_{N}} V_{i}(x) S(x)^{i}$, then Lemma 1 implies that $L_{N} \leq 4(N-1) D-2 D$ and $B_{N} \leq 2(N-2)\left(d_{S}^{f}-2 d_{S}^{h}\right)+$ $\left(d_{S}^{f}-d_{S}^{h}\right)$, with $d_{S}^{f}:=\operatorname{deg} f / \operatorname{deg} S$ and $d_{S}^{h}:=\operatorname{deg} h / \operatorname{deg} S$. Since we have to reduce the forms $x^{2 i+1} y^{\sigma} d x$ for $i=0, \ldots, 2 g-1$, the loss of precision will be determined by the reduction of $x^{4 g-1} V_{B_{N}}(x) S(x)^{B_{N}} y d x$ and $x V_{-L_{N}} S(x)^{-L_{N}} y d x$. The highest power of $x$ appearing in the former differential form is less than $2 N(\operatorname{deg} f-2 \operatorname{deg} h)+6 g$ and by Lemma 2 the loss of precision is bounded by $c_{N, 1}:=3+\left\lfloor\log _{2}(2 N(\operatorname{deg} f-2 \operatorname{deg} h)+7 g+1)\right\rfloor$. Similarly, Lemma 3 implies that the loss of precision introduced during the reduction of the latter differential form is bounded by $c_{N, 2}:=3+\left\lfloor\log _{2}(4 N D-6 D+1)\right\rfloor$. As a result, we conclude that it is sufficient to compute modulo $2^{N}$ with

$$
N>B+\max \left(c_{N, 1}, c_{N, 2}\right) .
$$




\section{Algorithm 1 (Hyperelliptic_Zeta_Function)}

IN: Hyperelliptic curve $\bar{C}$ over $\mathbb{F}_{q}$ given by equation $y^{2}+\bar{h}(x) y=\bar{f}(x)$. OUT: The zeta function $Z\left(\widetilde{C} / \mathbb{F}_{q} ; t\right)$.

1. $B=\left\lceil\log _{2}\left(2\left(\begin{array}{c}2 g \\ g\end{array}\right) q^{g / 2}\right)\right\rceil ; N>B+\max \left(c_{N, 1}, c_{N, 2}\right)$;

2. $(h(x), f(x), H(x), D)=$ Lift_Curve $(\bar{h}(x), \bar{f}(x))$;

3. $\alpha_{N}, \beta_{N}=$ Lift_Frobenius_y $(h, f, H, D, N)$;

4. For $i=0$ To $2 g-1$ Do

4.1. $R_{i}(x)=$ Reduce_MW_Cohomology $\left(2 x^{2 i+1} \beta_{N}, h, f, H, B\right)$;

4.2. For $j=0$ To $2 g-1$ Do $M[j][i]=\operatorname{Coeff}\left(R_{i}, j\right)$;

5. $M_{F}=M M^{\sigma} \cdots M^{\sigma^{n-1}} \bmod 2^{B}$;

6. $\chi(T)=$ Characteristic_Pol $\left(M_{F}\right) \bmod 2^{B}$;

7. For $i=0$ To $g$ Do

7.1. If Coeff $(\chi, 2 g-i)>\left(\begin{array}{c}2 g \\ i\end{array}\right) q^{i / 2}$ Then Coeff $(\chi, 2 g-i)-=2^{B}$;

7.2. $\operatorname{Coeff}(\chi, i)=q^{g-i} \operatorname{Coeff}(\chi, 2 g-i)$;

8. $\operatorname{Return} Z\left(\widetilde{C} / \mathbb{F}_{q} ; t\right)=\frac{t^{2 g} \chi(1 / t)}{(1-t)(1-q t)}$.

\subsection{Detailed Algorithm}

The function Hyperelliptic_Zeta_Function given in Algorithm 1 computes the zeta function of a smooth projective hyperelliptic curve $\widetilde{C}$ defined over $\mathbb{F}_{q}$ with $q=2^{n}$. Step 1 determines the minimal precision $N$ satisfying inequality (14).

In step 2 we call the subroutine Lift_Curve, which first constructs an isomorphic curve such that $\bar{H}(x) \mid \bar{h}(x)$ and $\bar{H}(x) \mid \bar{f}(x)$ and lifts the curve using the construction described in Section 3.2. The result of this function is a hyperelliptic curve $C: y^{2}+h(x) y=f(x)$ over $R$, a polynomial $H(x)$ and an integer $D$ such that $H(x)|h(x), H(x)| f(x)$ and $h(x) \mid H(x)^{D}$. Since this function is rather straightforward, we have omitted the pseudo-code.

In step 3 we compute $y^{\sigma}$ mod $2^{N}$ using the function Lift_Frobenius_y given in Algorithm 2. The parameters $\alpha_{N}, \beta_{N}$ are Laurent polynomials in $S$ with coefficients polynomials over $R \bmod 2^{N}$ of degree $<\operatorname{deg} S$. This function implements the Newton iteration (1), but has quadratic, instead of linear, convergence. Note that Algorithm 2 is in fact a double Newton iteration: $\alpha+\beta y$ converges to $y^{\sigma}$, whereas $\gamma+\delta y$ is an approximation of the inverse of the denominator (2) in the Newton iteration. 


\section{Algorithm 2 (Lift_Frobenius_y)}

IN: Curve $C: y^{2}+h(x) y=f(x)$ over $R$, polynomial $H(x) \in R[x]$ with $H \mid h$ and $H \mid f, D \in \mathbb{N}$ such that $h \mid H^{D}$ and precision $N$.

OUT: Laurent polynomials $\alpha_{N}, \beta_{N}$ in $S$ with $S=H$ if $\operatorname{deg} H>0$, $S=x$ if $H=1$ satisfying $y^{\sigma} \equiv \alpha_{N}+\beta_{N} y \bmod 2^{N}$.

1. $B=\left\lceil\log _{2} N\right\rceil+1 ; T=N ; Q_{S}:=S^{D} \operatorname{div} h$;

2. For $i=B$ Down To 1 Do $P[i]=T ; T=\lceil T / 2\rceil$;

3. $\alpha \equiv f \bmod 2 ; \beta \equiv-h \bmod 2 ; \gamma=1 ; \delta=0$;

4. For $i=2$ To $B$ Do

4.1. $T_{A} \equiv\left(\left(\alpha+h^{\sigma}\right) \cdot \alpha+\beta^{2} \cdot f-f^{\sigma}\right) \cdot Q_{S}^{2} \cdot S^{-2 D} \bmod 2^{P[i]}$;

4.2. $T_{B} \equiv\left(2 \alpha-h \cdot \beta+h^{\sigma}\right) \cdot \beta \cdot Q_{S}^{2} \cdot S^{-2 D} \bmod 2^{P[i]}$;

4.3. $D_{A} \equiv 1+\left(h^{\sigma}-h^{2}+2 \alpha\right) \cdot Q_{S}^{2} \cdot S^{-2 D} \bmod 2^{P[i-1]}$;

4.4. $D_{B} \equiv 2 \beta \cdot Q_{S}^{2} \cdot S^{-2 D} \bmod 2^{P[i-1]}$;

4.5. $V_{A} \equiv D_{A} \cdot \gamma+D_{B} \cdot \delta \cdot f-1 \bmod 2^{P[i-1]}$;

4.6. $V_{B} \equiv D_{A} \cdot \delta+D_{B} \cdot(\gamma-\delta \cdot h) \bmod 2^{P[i-1]}$;

4.7. $\gamma \equiv \gamma-\left(V_{A} \cdot \gamma+V_{B} \cdot \delta \cdot f\right) \bmod 2^{P[i-1]}$;

4.8. $\delta \equiv \delta-\left(V_{A} \cdot \delta+V_{B} \cdot(\gamma-\delta \cdot h)\right) \bmod 2^{P[i-1]}$;

4.9. $\alpha \equiv \alpha-\left(T_{A} \cdot \gamma+T_{B} \cdot \delta \cdot f\right) \bmod 2^{P[i]}$;

4.10. $\beta \equiv \beta-\left(T_{A} \cdot \delta+T_{B} \cdot(\gamma-\delta \cdot h)\right) \bmod 2^{P[i]}$;

5. Return $\alpha_{N}=\alpha, \beta_{N}=\beta$.

Once we have determined an approximation of $y^{\sigma}$, we compute the action of $\sigma_{*}$ on the basis of $H^{1}(\bar{A} / K)^{-}$as $2 x^{2 i+1} y^{\sigma} d x$ for $i=0, \ldots, 2 g-1$. In step 4 we reduce these forms using the function Reduce_MW_Cohomology given in Algorithm 3, which is based on the reduction formulae devised in Section 3.2. Given a differential $G y d x$ with $G$ a Laurent polynomial in $S$, this function computes a polynomial $\Lambda \in K[x]$, with $\operatorname{deg} \Lambda<2 g$ such that for a given precision $B$ we have the following equivalence modulo exact and invariant forms $\Lambda y d x \sim G y d x \bmod 2^{B}$, where mod $2^{B}$ means modulo $2^{B}\left(R y d x+\cdots+R x^{2 g-1} y d x\right)$. In step 2.3 we use the function XGCD which takes as input two polynomials $A(x), B(x) \in K[x]$ and returns polynomials $C(x), L_{A}(x), L_{B}(x)$ such that $C(x)=\operatorname{gcd}(A(x), B(x))$ and $C(x)=L_{A}(x) A(x)+L_{B}(x) B(x)$. Note that the remarks after Lemmata 2 and 3 imply that the result of Algorithm 3 is correct modulo $2^{B}$ since we computed modulo $2^{N}$ and $N$ satisfies $N-\max \left(c_{N, 1}, c_{N, 2}\right)>B$.

The result of step 4 of Algorithm 1 is an approximation modulo $2^{B}$ of the matrix $M$ through which $\sigma_{*}$ acts on $H^{1}(\bar{A} / K)^{-}$. In step 5 we compute its norm $M_{F}$ as $M M^{\sigma} \cdots M^{\sigma^{n-1}}$. 


\section{Algorithm 3 (Reduce_MW_Cohomology)}

IN: Polynomials $h(x), f(x), H(x) \in R[x]$ with $H \mid h$ and $H \mid f, H$ monic, Laurent polynomial $G=\sum T_{i}(x) S(x)^{i}$ with $S=H$ if $\operatorname{deg} H>0$ and $S=x$ if $H=1, T_{i}(x) \in R[x]$ with $\operatorname{deg} T_{i}<\operatorname{deg} S$, precisions $B, N$.

OUT: $\Lambda \in K[x]$, with $\operatorname{deg} \Lambda<2 g$ such that $\Lambda y d x \sim G y d x \bmod 2^{B}$.

1. $Q_{f}=f \operatorname{div} S ; Q_{h}=h \operatorname{div} S ; P=0 ; V=0 ; v_{G}=\operatorname{Valuation}(G)$;

2. For $i=v_{G}$ To -1

2.1. $V \equiv P+\operatorname{Coeff}(G, i) \bmod 2^{N}$;

2.2. $P \equiv V \operatorname{div} S \bmod 2^{N} ; V \equiv V-P \cdot S \bmod 2^{N}$;

2.3. $\left(1, L_{A}, L_{B}\right)=\operatorname{XGCD}\left(S, Q_{f} \cdot S^{\prime}\right)$;

2.4. $L_{A}=V \cdot L_{A} \bmod 2^{N} ; L_{B}=V \cdot L_{B} \bmod 2^{N}$;

2.5. $P \equiv P+L_{A}+\frac{L_{B} \cdot\left(-i Q_{h}^{2} \cdot S^{\prime}-3\left(2 Q_{f}^{\prime}+Q_{h} \cdot h^{\prime}\right)\right)-L_{B}^{\prime} \cdot\left(4 Q_{f}+Q_{h} h\right)}{6+4 i} \bmod 2^{N}$;

3. $d_{G}=\operatorname{Degree}(G) ; d_{T}=\left(d_{G}+1\right) \cdot \operatorname{Degree}(S) ; T=0$;

4. For $i=d_{G}$ Down To 0 Do $T=T \cdot S+\operatorname{Coeff}(G, i) \bmod 2^{N} ; T=T+P$;

5. For $i=d_{T}$ Down To $2 g$

5.1. $P \equiv x^{i-2 g}\left(2 f^{\prime}+h \cdot h^{\prime}\right)+\frac{i-2 g}{3} x^{i-2 g-1}\left(4 f+h^{2}\right) \bmod 2^{N}$;

5.2. $T \equiv T-(\operatorname{Coeff}(T, i) \cdot P) / \operatorname{Coeff}(P, i) \bmod 2^{N}$;

6. Return $\Lambda \equiv T \bmod 2^{B}$.

Note that since $M$ is not necessarily defined over $R$, we could lose up to $c n$ bits of precision, where $2^{c}$ is the largest denominator appearing in $M$. By Lemmata 1 and 2, $c$ is bounded by $O(\log g)$ independently of $n$. In theory we would therefore have to replace the bound $B$ in Algorithm 1 by $B+c n$, which does not change the complexity of the algorithm.

In practice however it turns out that the largest denominator appearing in $M_{F}$ is almost always the same as the largest denominator appearing in $M$ and therefore it is not necessary to increase $B$. This phenomenon can be heuristically explained as follows: since the eigenvalues of $F_{*}=\sigma_{*}^{n}$ on $H^{1}(\bar{A} / K)^{-}$have non-negative 2-adic valuation there is an $R$-submodule of $H^{1}(\bar{A} / K)^{-}$which is stable under the action of $\sigma_{*}$. For this $R$-submodule we can take for instance the canonical image of the crystalline cohomology of $C$ over $R$. Note that the $R$-submodule generated by $x^{i} y d x$ for $i=0, \ldots, 2 g-1$ is not canonical and in general not stable under $\sigma_{*}$. Let $A_{0}$ be the matrix that expresses $x^{i} y d x$ for $i=0, \ldots, 2 g-1$ in terms of a basis of such a stable $R$-submodule and let $A$ be $A_{0}$ times a power of 2 such that $A$ is a matrix over $R$ which is not zero modulo 2. Then $M=A^{-1} U A^{\sigma}$ where $U$ is the matrix of $\sigma_{*}$ with respect to the new basis. Note that $U$ is a matrix over $R$ and that the norm of $M$ equals 
$A^{-1} U U^{\sigma} \cdots U^{\sigma^{n-1}} A$. Thus the loss of precision is no more than $d$ bits where $d$ is the 2 -adic valuation of $\operatorname{det}(A)$. If $U$ and $A$ are generic enough then $|c-d|$ is small. Furthermore, the bound (14) turns out to be sligthly larger than what is needed and compensates for the loss of $d$ bits.

In steps 6 and 7 we recover the characteristic polynomial of Frobenius from the first $g$ coefficients of the characteristic polynomial of $M_{F}$. Finally, we return the zeta function of the smooth projective hyperelliptic curve $\widetilde{C}$ birational to $\bar{C}$ in step 8.

\subsection{Complexity}

In this section we analyze the space and time requirements of Algorithm 1 for a genus $g$ hyperelliptic curve over $\mathbb{F}_{2^{n}}$ assuming fast arithmetic, i.e. using the Schönhage-Strassen algorithm [35] that computes the product of two $m$-bit integers in time $O\left(m^{1+\varepsilon}\right)$ for any constant $\varepsilon \in \mathbb{R}_{>0}$. Before proceeding through the individual steps of the algorithm, we analyze the complexity of the basic operations in Algorithm 1 and the asymptotic behaviour of the bounds given in Lemma 1.

For a fixed precision $N$, let $R_{N}$ denote the degree $n$ unramified extension of $\mathbb{Z}_{2} / 2^{N} \mathbb{Z}_{2}$. Elements of $R_{N}$ are represented as polynomials over $\mathbb{Z} / 2^{N} \mathbb{Z}$ modulo a sparse irreducible polynomial $P(t)$ of degree $n$. Since each element of this ring requires $O(n N)$ space, we can perform the basic operations, i.e. multiplication and division, in time $O\left(n^{1+\varepsilon} N^{1+\varepsilon}\right)$.

Computing the Frobenius substitution $\sigma$ on $R_{N}$ can be accomplished in time $O\left(n^{2+\varepsilon} N^{1+\varepsilon}\right)$ as follows. Since $t$ is a root of $P(t), t^{\sigma}$ will also be a root of $P(t)$ and $t^{\sigma} \equiv t^{2} \bmod 2$. Therefore, $t^{\sigma}$ can be computed using the Newton iteration $T_{k+1}=$ $T_{k}-P\left(T_{k}\right) / P^{\prime}\left(T_{k}\right)$ initialized with $t^{2}$. Since the Newton iteration converges quadratically and we compute with the minimal precision in each step, the total complexity will be determined by the last step which takes $O(n)$ multiplications in $R_{N}$. Precomputing $t^{\sigma} \bmod 2^{N}$ can thus be accomplished in time $O\left(n^{2+\varepsilon} N^{1+\varepsilon}\right)$. After this precomputation, we can compute the Frobenius substitution of any element $E(t)$ as $E\left(t^{\sigma}\right)$, which needs $O(n)$ multiplications in $R_{N}$ and thus takes $O\left(n^{2+\varepsilon} N^{1+\varepsilon}\right)$ time.

Lemma 1 bounds the maximum bit-size of the Laurent series we compute with and therefore determines the complexity of Algorithm 1. Since these bounds depend on the degree and splitting type of $h(x)$, we make a distinction between average-case and worst-case complexity. To this end we introduce three parameters which allow us to analyze both cases simultaneously.

- Let the asymptotic behaviour of $\operatorname{deg} f-2 \operatorname{deg} h$ be $O\left(g^{\lambda}\right)$. Since the degree of $f(x)$ is $2 g+1$ and $h(x)$ is a random polynomial of degree $\leq g$, we conclude that $\lambda=0$ on average and $\lambda=1$ in the worst case.

- Let the asymptotic behaviour of $\operatorname{deg} H$ be $O\left(g^{\mu}\right)$. With very high probability a random polynomial of degree $\leq g$ has $O(g)$ different roots, which implies that $\mu=1$ on average and $\mu=0$ in the extreme case. 
- Let the asymptotic behaviour of $D$ be $O\left(g^{\nu}\right)$, then $\nu=0$ on average and $\nu=1$ in the worst case, since with very high probability a random polynomial only has roots with multiplicity $O(1)$.

In step 1 of Algorithm 1 we determine the minimal precision $N$ satisfying inequality (14), which implies that $N$ is $O(g n)$. The function Lift_Frobenius_y in step 3 is a Newton lifting. Since the precision doubles in every iteration, we see that its complexity is determined by the last iteration, which consists of $O(1)$ multiplications of Laurent polynomials in $S$ with coefficients polynomials over $R_{N}$ of degree less than $\operatorname{deg} S$. Lemma 1 implies that the bit-size of these objects is $O\left(\left(g^{\lambda}+g^{\mu+\nu}\right) n N^{2}\right)$. Since the cost of the other operations in Lift_Frobenius_y, e.g. computing the Frobenius substitution of $O(g)$ elements of $R_{N}$, is less than the $O(1)$ multiplications, the overall time complexity of step 3 is $O\left(\left(g^{\lambda}+g^{\mu+\nu}\right)^{1+\varepsilon} n^{1+\varepsilon} N^{2+\varepsilon}\right)$.

In step 4 of Algorithm 1 we reduce the $2 g$ differential forms $2 x^{2 i+1} \beta_{N} y d x$ for $i=0, \ldots, 2 g-1$ using the function Reduce_MW_Cohomology given in Algorithm 3. In step 3 the dominant operations are $O(1)$ multiplications of polynomials over $R_{N}$ of degree $O(g)$ and the extended GCD computation of two such polynomials. The former operation clearly takes time $O\left(g^{1+\varepsilon} n^{1+\varepsilon} N^{1+\varepsilon}\right)$ and using Moenck's algorithm [28] the latter operation can also be performed in time $O\left(g^{1+\varepsilon} n^{1+\varepsilon} N^{1+\varepsilon}\right)$. Lemma 1 implies that these operations have to be repeated $O\left(g^{\nu} N\right)$ times, so the time complexity of step 3 is $O\left(g^{1+\nu+\varepsilon} n^{1+\varepsilon} N^{2+\varepsilon}\right)$. Write $\beta_{N}$ as $\sum_{i=-L_{N}}^{B_{N}} V_{i}(x) S(x)^{i}$, then step 5 essentially is Horner's rule to compute $\sum_{i=0}^{B_{N}} V_{i}(x) S(x)^{i}$. Note that in practice we perform this step only once for all of the $2 g$ reductions and use a binary tree algorithm which is asymptotically faster than Horner's method. The complexity of step 5 then becomes $O\left(g^{\lambda+\varepsilon} n^{1+\varepsilon} N^{2+\varepsilon}\right)$. Lemma 1 implies that substeps 6.1 and 6.2 have to be executed $O\left(g^{\lambda} N\right)$ times and since each iteration consists of $O(g)$ multiplications and $O(1)$ divisions in $R_{N}$, the time complexity of step 6 is $O\left(g^{1+\lambda} n^{1+\varepsilon} N^{2+\varepsilon}\right)$. Since we have to reduce $O(g)$ differential forms, the overall time complexity of step 4 of Algorithm 1 is $O\left(\left(g^{2+\lambda}+g^{2+\nu+\varepsilon}\right) n^{1+\varepsilon} N^{2+\varepsilon}\right)$.

In step 5 we need to determine the norm of a $2 g \times 2 g$ matrix $M$ over $K$ as $M M^{\sigma} \cdots M^{\sigma^{n-1}}$. This can be accomplished by computing $M_{i+1}=M_{i} M_{i}^{\sigma^{2^{i}}}$ for $i=0, \ldots,\left\lfloor\log _{2} n\right\rfloor$ with $M_{0}=M$ and combining these to recover the norm of $M$. This process takes $O(\log n)$ multiplications of $2 g \times 2 g$ matrices at a cost of $O\left(g^{3} n^{1+\varepsilon} N^{1+\varepsilon}\right)$ time and $O\left(g^{2} \log n\right)$ applications of powers of $\sigma$ which takes $O\left(g^{2} n^{2+\varepsilon} N^{1+\varepsilon}\right)$ time if we precompute $t^{\sigma^{2^{i}}}$ for $i=0, \ldots,\left\lfloor\log _{2} n\right\rfloor$. The overall time complexity of step 5 thus becomes $O\left((n+g) g^{2} n^{1+\varepsilon} N^{1+\varepsilon}\right)$.

Finally, we need to compute the characteristic polynomial of a $2 g \times 2 g$ matrix over $K$, which can be done using the classical algorithm based on the Hessenberg form [6, Section 2.2.4]. The complexity of this algorithm is $O\left(g^{3}\right)$ ring operations or $O\left(g^{3} n^{1+\varepsilon} N^{1+\varepsilon}\right)$ time.

Since equation (14) implies that $N$ is $O(g n)$, the overall time complexity of Algorithm 1 is $O\left(\left(g^{\lambda}+g^{\nu}\right) g^{4+\varepsilon} n^{3+\varepsilon}\right)$ and the overall space complexity becomes $O\left(\left(g^{\lambda}+g^{\mu+\nu}\right) g^{2} n^{3}\right)$. Note that this means the following: 
- Average case: the time complexity reduces to $O\left(g^{4+\varepsilon} n^{3+\varepsilon}\right)$ and the space complexity is $O\left(g^{3} n^{3}\right)$.

- Worst case: the time complexity grows to $O\left(g^{5+\varepsilon} n^{3+\varepsilon}\right)$ and the space complexity becomes $O\left(g^{4} n^{3}\right)$.

\section{Implementation and Numerical Results}

In this section we present running times of an implementation of Algorithm 1 in the C programming language and give some examples of Jacobians of hyperelliptic curves with almost prime group order.

The basic operations on integers modulo $2^{N}$ for $N \leq 256$ were written in assembly language. Elements of $R_{N}$ are represented as polynomials over $\mathbb{Z} / 2^{N} \mathbb{Z}$ modulo a degree $n$ irreducible polynomial, which we chose to be either a trinomial or a pentanomial. For multiplication of elements in $R_{N}$, polynomials over $R_{N}$ and Laurent series over $R_{N}[x]$ we used Karatsuba's trick [16], which allows to multiply two $m$-bit integers in time $O\left(m^{\log _{2} 3}\right)$. Redoing the complexity analysis then results in an average-case time complexity of $O\left(n^{4.75} g^{5.17}\right)$ bit-operations.

\subsection{Running Times and Memory Usage}

Table 1 contains running times and memory usages of our algorithm for genus 2, 3 and 4 hyperelliptic curves over various finite fields of characteristic 2 . These results were obtained on an AMD XP 1700+ processor running Linux Redhat 7.1. Note that the field degrees are chosen such that $g n$, and therefore the size of the group order of the Jacobian, is constant across each row.

Table 1. Running time (s) and memory usage (MB) for genus 2, 3 and 4 hyperelliptic curves over $\mathbb{F}_{2^{n}}$

\begin{tabular}{|c||c|c||c|c||c|c|}
\hline Size of Jacobian & \multicolumn{2}{|c||}{ Genus 2 curves } & \multicolumn{2}{c||}{ Genus 3 curves } & \multicolumn{2}{c|}{ Genus 4 curves } \\
\hline$g n$ & Time (s) & Mem (MB) & Time (s) & Mem (MB) & Time (s) & Mem (MB) \\
\hline 120 & 22 & 4.5 & 28 & 5.4 & 26 & 5.2 \\
144 & 35 & 5.7 & 46 & 7.3 & 43 & 7.2 \\
168 & 60 & 8.6 & 78 & 11 & 76 & 11 \\
192 & 89 & 13 & 112 & 14 & 109 & 13 \\
216 & 143 & 16 & 171 & 17 & 157 & 16 \\
\hline
\end{tabular}

\subsection{Hyperelliptic Curve Examples}

In this subsection we give three examples of Jacobians of hyperelliptic curves with almost prime group order. The correctness of these results is easily proved by multiplying a random divisor with the given group order and verifying that the result is principal, i.e. is the zero element in the Jacobian $J_{\widetilde{C}}\left(\mathbb{F}_{q}\right)$. It is clear that the given curves are non-supersingular, since the coefficient $a_{g}$ of $\chi(T)$ is odd [11]. Let $\alpha=\sum_{i=0}^{n-1} \alpha_{i} t^{i} \in \mathbb{F}_{2^{n}}$, then $\alpha$ is represented by the integer $\sum_{i=0}^{n-1} \alpha_{i} 2^{i}$ written in hexadecimal notation. 


\section{Genus 2 hyperelliptic curve over $\mathbb{F}_{2^{83}}$}

Let $\mathbb{F}_{283}$ be defined as $\mathbb{F}_{2}[t] / \bar{P}(t)$ with $\bar{P}(t)=t^{83}+t^{7}+t^{4}+t^{2}+1$ and consider the random hyperelliptic curve $C_{2}$ of genus 2 defined by

$$
y^{2}+\left(\sum_{i=0}^{2} h_{i} x^{i}\right) y=x^{5}+\sum_{i=0}^{4} f_{i} x^{i}
$$

where

$$
\begin{array}{ll}
h_{0}=\text { 4D168CAB78F1F7EB78D54 } & h_{1}=3 \text { B167A2F520486B2A8A60 } \\
h_{2}=507 F C 6 \text { DD98A1411D1F24 } & \\
f_{0}=\text { 6ABF379716E615F0997AF } & f_{1}=\text { 1D13C5C10A58A238681F3 } \\
f_{2}=\text { 3ACC287DAA28D01EDDB58 } & f_{3}=74 \text { BF8FFD1A04B1E8B845B } \\
f_{4}=10046 \text { AOED36CF3B146071 } &
\end{array}
$$

The group order of the Jacobian $J_{\widetilde{C}_{2}}$ of $C_{2}$ over $\mathbb{F}_{2^{83}}$ is

$$
\# J_{\widetilde{C}_{2}}=2 \cdot 46768052394612054553468807679365619497317916118893,
$$

where the last factor is prime. The coefficients $a_{1}$ and $a_{2}$ of the characteristic polynomial of Frobenius $\chi(T)=T^{4}+a_{1} T^{3}+a_{2} T^{2}+a_{3} T+a_{4}$ are given by

$$
a_{1}=4789617893650 \text { and } a_{2}=12304549269471460402134471 .
$$

\section{Genus 3 hyperelliptic curve over $\mathbb{F}_{2^{59}}$}

Let $\mathbb{F}_{2^{59}}$ be defined as $\mathbb{F}_{2}[t] / \bar{P}(t)$ with $\bar{P}(t)=t^{59}+t^{7}+t^{4}+t^{2}+1$ and consider the random hyperelliptic curve $C_{3}$ of genus 3 defined by

$$
y^{2}+\left(\sum_{i=0}^{3} h_{i} x^{i}\right) y=x^{7}+\sum_{i=0}^{6} f_{i} x^{i}
$$

where

$$
\begin{array}{ll}
h_{0}=44 \mathrm{ECOA3F607D5FE} & h_{1}=183 \mathrm{AFFC60B6C97A} \\
h_{2}=\text { 5E8C286F052173E } & h_{3}=39 \mathrm{BFF} 4 \mathrm{C} 327 \mathrm{D} 0 \mathrm{FCC} \\
f_{0}=2 \text { CE03A6BD01418F } & f_{1}=15160 \text { EE501EA31D } \\
f_{2}=2 \text { DDF3B805A56673 } & f_{3}=72 \mathrm{EAAC2B03D6F33} \\
f_{4}=30 \mathrm{BF} 8 \mathrm{CAF} 4 \mathrm{CF} 398 \mathrm{~A} & f_{5}=288 \mathrm{~F} 45 \mathrm{CEB} 700047 \\
f_{6}=692 \mathrm{BDF} 3913214 \mathrm{~F} 7 &
\end{array}
$$

The group order of the Jacobian $J_{\widetilde{C}_{3}}$ of $C_{3}$ over $\mathbb{F}_{2^{59}}$ is

$$
\text { \# } J_{\widetilde{C}_{3}}=2 \cdot 95780971232851005943503002779523943538413536699032693 \text {, }
$$

where the last factor is prime. The coefficients $a_{1}, a_{2}$ and $a_{3}$ of the characteristic polynomial of Frobenius $\chi(T)=T^{6}+a_{1} T^{5}+a_{2} T^{4}+a_{3} T^{3}+a_{4} T^{2}+a_{5} T+a_{6}$ are given by

$$
\begin{aligned}
& a_{1}=-428922942, \\
& a_{2}=394510910624097420, \\
& a_{3}=-307916874056151778020344677 .
\end{aligned}
$$




\section{Genus 4 hyperelliptic curve over $\mathbb{F}_{2^{47}}$}

Let $\mathbb{F}_{2^{47}}$ be defined as $\mathbb{F}_{2}[t] / \bar{P}(t)$ with $\bar{P}(t)=t^{47}+t^{5}+1$ and consider the random hyperelliptic curve $C_{4}$ of genus 4 defined by

$$
y^{2}+\left(\sum_{i=0}^{4} h_{i} x^{i}\right) y=x^{9}+\sum_{i=0}^{8} f_{i} x^{i}
$$

where

$$
\begin{array}{lll}
h_{0}=45 \text { EDAA69BB7B } & h_{1}=29185 \mathrm{CC} 987 \mathrm{~F} 2 & h_{2}=\text { 5B56AF467634 } \\
h_{3}=063 \mathrm{~A} 420 \mathrm{D} 7308 & h_{4}=3 \text { AD67360D2FB } & \\
f_{0}=116 \mathrm{~A} 64 \mathrm{DA4E4A} & f_{1}=1267 \mathrm{C} 8 \mathrm{BFEDF} 4 & f_{2}=5 \mathrm{DED} 53867285 \\
f_{3}=3 \text { E2486D3500B } & f_{4}=66718 \mathrm{C} 5 \mathrm{D} 41 \mathrm{BD} & f_{5}=5 \text { FBD515320F } 1 \\
f_{6}=4 \mathrm{~B} 960757 \mathrm{EC} 52 & f_{7}=67 \mathrm{~B} 0202 \mathrm{BA} 7 \mathrm{D} 5 & f_{8}=545283 \mathrm{~F} 149 \mathrm{~A} 8
\end{array}
$$

The group order of the Jacobian $J_{\widetilde{C}_{4}}$ of $C_{4}$ over $\mathbb{F}_{2^{43}}$ is

$$
\# J_{\widetilde{C}_{4}}=2 \cdot 196159429641733316151830117421270924231809135724223902787 \text {, }
$$

where the last factor is prime. The coefficients $a_{1}, a_{2}, a_{3}$ and $a_{4}$ of the characteristic polynomial of Frobenius $\chi(T)=T^{8}+a_{1} T^{7}+a_{2} T^{6}+a_{3} T^{5}+a_{4} T^{4}+$ $a_{5} T^{3}+a_{6} T^{2}+a_{7} T+a_{8}$ are given by

$$
\begin{aligned}
& a_{1}=294806 \\
& a_{2}=-5127513198846, \\
& a_{3}=236526738819576049756, \\
& a_{4}=31534922966327446198018115985 .
\end{aligned}
$$

\section{Conclusion}

In this paper we have presented an extension of Kedlaya's algorithm to compute the zeta function of an arbitrary hyperelliptic curve $C$ over a finite field of characteristic 2. The main difference with Kedlaya's algorithm is that the hyperelliptic curve can no longer be lifted arbitrarily; instead, a very specific lift is needed to ensure that the algebraic de Rham cohomology and the MonskyWashnitzer cohomology are isomorphic. For a genus $g$ hyperelliptic curve defined over $\mathbb{F}_{2^{n}}$, the average-case time complexity is $O\left(g^{4+\varepsilon} n^{3+\varepsilon}\right)$ and the average-case space complexity is $O\left(g^{3} n^{3}\right)$, whereas the worst-case time and space complexities are $O\left(g^{5+\varepsilon} n^{3+\varepsilon}\right)$ and $O\left(g^{4} n^{3}\right)$ respectively. An implementation in the C programming language shows that cryptographical sizes are now feasible for any genus $g$, e.g. computing the order of a 160-bit Jacobian of a hyperelliptic curve of genus 2, 3 or 4 takes about 75 seconds. Due to the generality of the cohomological approach, it seems likely that Kedlaya's algorithm can be extended to arbitrary curves. For a first step in this direction, we refer to [8] which presents an algorithm to compute the zeta function of any non-singular $C_{a b}$ curve over a finite field of small characteristic. 


\section{References}

1. L. M. Adleman and M.-D. Huang. Counting rational points on curves and abelian varieties over finite fields. In H. Cohen, editor, Algorithmic number theory. 2nd international symposium. ANTS-II, volume 1122 of Lecture Notes in Computer Science, pages 1-16, 1996.

2. S. Arita. Algorithms for computations in Jacobians of $C_{a b}$ curves and their application to discrete-log-based public key cryptosystems. In Proceedings of Conference on The Mathematics of Public Key Cryptography, 1999.

3. A.O.L. Atkin. The number of points on an elliptic curve modulo a prime. Series of e-mails to the NMBRTHRY mailing list, 1992.

4. I.F. Blake, G. Seroussi, and N.P. Smart. Elliptic curves in cryptography. London Mathematical Society Lecture Note Series. 265. Cambridge University Press., 1999.

5. S. Bosch. A rigid analytic version of M. Artin's theorem on analytic equations. Math. Ann., 255:395-404, 1981.

6. H. Cohen. A course in computational algebraic number theory. Graduate Texts in Mathematics. 138. Springer-Verlag., 1993.

7. J. Denef and F. Vercauteren. An extension of Kedlaya's algorithm to Artin-Schreier curves in characteristic 2. In C. Fieker and D.R. Kohel, editors, Algorithmic number theory. 5th international symposium. ANTS-V, volume 2369 of Lecture Notes in Computer Science, pages 308-323, 2002.

8. J. Denef and F. Vercauteren. Counting points on $C_{a b}$ curves using MonskyWashnitzer cohomology. Available at http://www.cs.bris.ac.uk/ frederik/, 2003.

9. N. Elkies. Elliptic and modular curves over finite fields and related computational issues. Computational Perspectives on Number Theory, pages 21-76, 1998.

10. R. Elkik. Solutions d'équations a coefficients dans un anneau henselien. Ann. Scient. Ec. Norm. Syp., 6(4):553-604, 1973.

11. S. Galbraith. Supersingular curves in cryptography. In C. Boyd, editor, Advances in Cryptology - ASIACRYPT 2001, volume 2248 of Lecture Notes in Computer Science, pages 495-513, 2001.

12. S. Galbraith, S. Paulus, and N. Smart. Arithmetic on superelliptic curves. Math. Comp., 71(237):393-405, 2002.

13. P. Gaudry and N. Gürel. An extension of Kedlaya's algorithm for counting points on superelliptic curves. In C. Boyd, editor, Advances in Cryptology - ASIACRYPT 2001, volume 2248 of Lecture Notes in Computer Science, pages 480-494, 2001.

14. P. Gaudry and R. Harley. Counting points on hyperelliptic curves over finite fields. In Wieb Bosma, editor, Algorithmic number theory. 4th international symposium. ANTS-IV, volume 1838 of Lecture Notes in Computer Science, pages 313-332, 2000.

15. P. Gaudry and É. Schost Construction of secure random curves of genus 2 over prime fields. In C. Cachin and J. Camenisch, editors, Advances in Cryptology EUROCRYPT 2004, volume 3027 of Lecture Notes in Computer Science, pages 239-256, 2004.

16. A. Karatsuba and Y. Ofman. Multiplication of multidigit numbers on automata. Soviet Physics Doklady, 7:595-596, 1963.

17. K.S. Kedlaya. Counting points on hyperelliptic curves using Monsky-Washnitzer cohomology. J. Ramanujan Math. Soc., 16:323-338, 2001.

18. N. Koblitz. Elliptic curve cryptosystems. Math. Comp., 48(177):203-209, 1987.

19. N. Koblitz. Hyperelliptic cryptosystems. J. Cryptology, 1(3):139-150, 1989. 
20. A. Lauder and Wan. D. Counting points on varieties over finite fields of small characteristic. In J.P. Buhler and P. Stevenhagen, editors, Algorithmic Number Theory: Lattices, Number Fields, Curves and Cryptography, Mathematical Sciences Research Institute Publications, 2002. To appear.

21. A. G. B. Lauder and D. Wan. Computing zeta functions of Artin-Schreier curves over finite fields. LMS J. Comput. Math., 5:34-55 (electronic), 2002.

22. A.G.B. Lauder and D. Wan. Computing zeta functions of Artin-Schreier curves over finite fields II. Journal of Complexity, 20:331-349, 2004.

23. R. Lercier. Algorithmique des courbes elliptiques dans les corps finis. $\mathrm{PhD}$ thesis, Laboratoire d'Informatique de l'École polytechnique (LIX), 1997.

24. R. Lercier and D. Lubicz. A quasi quadratic time algorithm for hyperelliptic curve point counting. Preprint 2003.

25. J.-F. Mestre. Lettre adressée à Gaudry et Harley, December 2000. Available at http://www.math.jussieu.fr/ mestre/.

26. J.-F. Mestre. Algorithmes pour compter des points en petite caractéristique en genre 1 and 2. Available at http://www. math. univ-rennes1.fr/crypto/2001-02/ mestre.ps.

27. V. S. Miller. Use of elliptic curves in cryptography. In H. C. Williams, editor, Advances in Cryptology - CRYPTO 1985, volume 218 of Lecture Notes in Computer Science, pages 417-426, 1986.

28. R.T. Moenck. Fast computation of GCDs. Fifth Annual ACM Symposium on Theory of Computing, pages 142-151, 1973.

29. P. Monsky and G. Washnitzer. Formal cohomology. I. Ann. of Math., 88:181-217, 1968.

30. P. Monsky. Formal cohomology. II: The cohomology sequence of a pair. Ann. of Math., 88:218-238, 1968.

31. P. Monsky. Formal cohomology. III: Fixed point theorems. Ann. of Math., 93:315343, 1971.

32. P. Monsky. p-adic analysis and zeta functions. Lectures in Mathematics, Department of Mathematics Kyoto University. 4. Tokyo, Japan, 1970.

33. J. Pila. Frobenius maps of abelian varieties and finding roots of unity in finite fields. Math. Comp., 55(192):745-763, 1990.

34. T. Satoh. The canonical lift of an ordinary elliptic curve over a finite field and its point counting. J. Ramanujan Math. Soc., 15:247-270, 2000.

35. A. Schönhage and V. Strassen. Schnelle Multiplikation grosser Zahlen. Computing (Arch. Elektron. Rechnen), 7:281-292, 1971.

36. R. Schoof. Elliptic curves over finite fields and the computation of square roots $\bmod$ p. Math. Comp., 44(170):483-494, 1985.

37. M. van der Put. The cohomology of Monsky and Washnitzer. Mém. Soc. Math. France, 23:33-60, 1986.

38. F. Vercauteren. Computing Zeta Functions of Hyperelliptic Curves over Finite Fields of Characteristic 2. In M. Yung, editor, Advances in Cryptology - CRYPTO 2002, volume 2442 of Lecture Notes in Computer Science, pages 369-384, 2002.

39. F. Vercauteren. Computing Zeta Functions of Curves over Finite Fields. PhD thesis, Katholieke Universiteit Leuven, November 2003. Available at http://www . cs.bris.ac.uk/ frederik/. 